\title{
SPECTRAL INTEGRATION AND SPECTRAL THEORY FOR NON-ARCHIMEDEAN BANACH SPACES
}

\author{
S. LUDKOVSKY and B. DIARRA
}

Received 22 January 2001 and in revised form 8 August 2001

\begin{abstract}
Banach algebras over arbitrary complete non-Archimedean fields are considered such that operators may be nonanalytic. There are different types of Banach spaces over nonArchimedean fields. We have determined the spectrum of some closed commutative subalgebras of the Banach algebra $\mathscr{L}(E)$ of the continuous linear operators on a free Banach space $E$ generated by projectors. We investigate the spectral integration of nonArchimedean Banach algebras. We define a spectral measure and prove several properties. We prove the non-Archimedean analog of Stone theorem. It also contains the case of $C$-algebras $C_{\infty}(X, \mathbb{K})$. We prove a particular case of a representation of a $C$-algebra with the help of a $L(\hat{A}, \mu, \mathbb{K})$-projection-valued measure. We consider spectral theorems for operators and families of commuting linear continuous operators on the non-Archimedean Banach space.
\end{abstract}

2000 Mathematics Subject Classification: 47A10, 47A25, 47L10.

1. Introduction. This paper is devoted to the non-Archimedean theory of spectral integration with the help of the projection-valued measure. Spectral integration plays a very important role in the theory of Banach algebras, theory of operators and has applications to the representation theory of groups and algebras in the classical case of the field of complex numbers $\mathbb{C}[7,8,14,13,19,23]$. There are also several works about non-Archimedean Banach algebra theory, which show that there are substantial differences between the non-Archimedean and classical cases [3, 5, 6, $10,11,12,18,26,27,28,30]$. In $[3,30]$, analytic operators over $\mathbb{C}_{p}$ were considered and the Shnirelman integration of analytic functions was used, which differs strongly from the non-Archimedean integration theory related to the measure theory [28]. In the non-Archimedean case, the spectral theory differs from the classical results of Gelfand-Mazur, because quotients of commutative Banach algebras over a field $\mathbb{K}$ by maximal ideals may be fields $\mathbb{E}$, which contain $\mathbb{K}$ as a proper subfield [28]. In general for each non-Archimedean field $\mathbb{K}$, there exists its extension $\mathbb{F}$ such that a field $\mathbb{E} \neq \mathbb{K}$ $[4,25]$.

Ideals and maximal ideals of non-Archimedean commutative $E$-algebras (see Section 5.1.1) and $C$-algebras were investigated in [28, 29]. In [5, 6], it was shown that the failure of the spectral theory in the non-Archimedean analog of the Hilbert space and it was shown that even symmetry properties of matrices lead to the enlargement of the initial field while a diagonalisation procedure. In [10, 11, 12], formulas of the spectral radius and different notions of spectrum and analysed some aspects of structures of non-Archimedean Banach algebras. In [28] and the references therein, general theory 
of non-Archimedean Banach algebras and their isomorphisms was considered. It was introduced the notion of $C$-algebras in the non-Archimedean case apart from the classical $C^{*}$-algebras. There are principal differences in the orthogonality in the Hilbert space over $\mathbb{C}$ and orthogonality in the non-Archimedean Banach space. Therefore, symmetry properties of operators do not play the same role in the non-Archimedean case as in the classical case.

This paper treats another aspect of the non-Archimedean algebra theory and theory of operators. Banach algebras over arbitrary complete non-Archimedean fields are considered such that the operators may be nonanalytic. There are different types of Banach spaces over non-Archimedean fields. In Sections 2, 3, and 4, are considered specific spaces. In Section 5, are considered general cases.

Let $\mathbb{K}$ be a field. A non-Archimedean valuation on $\mathbb{K}$ is a function $|*|: \mathbb{K} \rightarrow \mathbb{R}$ such that

(1) $|x| \geq 0$ for each $x \in \mathbb{K}$;

(2) $|x|=0$ if and only if $x=0$;

(3) $|x+y| \leq \max (|x|,|y|)$ for each $x$ and $y \in \mathbb{K}$;

(4) $|x y|=|x||y|$ for each $x$ and $y \in \mathbb{K}$.

The field $\mathbb{K}$ is called topologically complete if it is complete relative to the following metric: $\rho(x, y)=|x-y|$ for each $x$ and $y \in \mathbb{K}$. A topological vector space $E$ over $\mathbb{K}$ with the non-Archimedean valuation may have a norm $\|*\|$ such that its restriction on each one-dimensional subspace over $\mathbb{K}$ coincides with the valuation $|*|$. If $E$ is complete relative to such norm $\|*\|$, then it is called the Banach space. Such fields and topological vector spaces are called non-Archimedean. An algebra $X$ over $\mathbb{K}$ is called Banach, if it is a Banach space as a topological vector space and the multiplication in it is continuous such that $\|x y\| \leq\|x\|\|y\|$ for each $x$ and $y$ in $X$. A finite or infinite sequence $\left(x_{j}: j \in \Lambda\right.$ ) of elements in a normed space $E$ is called orthogonal, if $\left\|\sum_{j \in \Lambda} \alpha_{j} x_{j}\right\|=\max \left(\left\|\alpha_{j} x_{j}\right\|: j \in \Lambda\right)$ for each $\alpha_{j} \in \mathbb{K}$ for which $\lim _{j} \alpha_{j} x_{j}=0$. We consider the infinite topologically complete field $\mathbb{K}$ with the nontrivial non-Archimedean valuation.

A non-Archimedean Banach space $E$ is said to be free if there exists a family $\left(e_{j}\right.$ : $j \in I) \subset E$ such that any element $x \in E$ can be written in the form of convergent sum $x=\sum_{j \in I} x_{j} e_{j}$, that is, $\lim _{j \in I} x_{j} e_{j}=0$ and $\|x\|=\sup _{j \in I}\left|x_{j}\right|\left\|e_{j}\right\|$ (see Section 2). In Section 3, ultrametric Hilbert spaces are considered. In Section 4, we have determined the spectrum of some closed commutative subalgebras of the Banach algebra $\mathscr{L}(E)$ of the continuous linear operators of $E$ generated by projectors.

Section 5 is devoted to the spectral integration. We introduce another definition of $E$-algebras in Section 5.1 apart from [29]. In Propositions 5.2 and 5.3 we have proved that they are contained in the class of $E$-algebras and $C$-algebras considered in [28, 29]. In Section 5.2, a spectral measure is defined. In Section 5.5, Lemma 5.5, Corollary 5.6, Proposition 5.7, and Corollary 5.9 its several properties are proved. In Theorem 5.11 the non-Archimedean analog of Stone theorem is proved. It contains also the case of $C$-algebras $C_{\infty}(X, \mathbb{K})$. A particular case of a representation of a $C$-algebra with the help of $L(\hat{A}, \mu, \mathbb{K})$-projection-valued measure is proved in Theorem 5.14. Spectral theorems for operators and families of commuting linear continuous operators on a non-Archimedean Banach space are considered in Sections 5.8 and 5.9. 


\section{Free Banach spaces}

2.1. Let $E$ be a free Banach space with an orthogonal base $\left(e_{j}: j \in I\right)$. The topological dual $E^{\prime}$ of $E$ is a Banach space with respect to the norm defined for $x^{\prime} \in E^{\prime}$ by $\left\|x^{\prime}\right\|=\sup _{x \neq 0}\left|\left\langle x^{\prime}, x\right\rangle\right| /\|x\|$. For $x^{\prime} \in E^{\prime}$ and $y \in E$, we define an element $\left(x^{\prime} \otimes y\right)$ of the Banach algebra of continuous linear operators $\mathscr{L}(E)$ on the space $E$ by setting for $x \in E,\left(x^{\prime} \otimes y\right)(x)=\left\langle x^{\prime}, x\right\rangle y$ with norm $\left\|x^{\prime} \otimes y\right\|=\left\|x^{\prime}\right\|\|y\|$. If $E$ is a free Banach space with base $\left(e_{j}: j \in I\right)$, any $u \in \mathscr{L}(E)$ can be written as a pointwise convergent sum $u=\sum_{(i, j) \in I \times I} \alpha_{i j} e_{j}^{\prime} \otimes e_{i}$. Hence $\lim _{i \in I} \alpha_{i j} e_{i}=0$ for each $j \in I$. Moreover $\|u\|=\sup _{i, j}\left|\alpha_{i j}\right|\left\|e_{j}^{\prime}\right\|\left\|e_{i}\right\|$. Notice that $\left\|e_{j}^{\prime}\right\|=1 /\left\|e_{j}\right\|$. Let $\mathscr{L}_{0}(E)=\{u: u=$ $\sum_{(i, j) \in I \times I} \alpha_{i j} e_{j}^{\prime} \otimes e_{i} \in \mathscr{L}(E) ; \lim _{j \in I} \alpha_{i j} e_{j}^{\prime}=0$ for each $\left.i \in I\right\}$.

THEOREM 2.1. An algebra $\mathscr{L}_{0}(E)$ is a closed subalgebra in $\mathscr{L}(E)$ with the unit element of $\mathscr{L}(E)$.

Proof. Let $u, v \in \mathscr{L}_{0}(E), u=\sum_{(i, j) \in I \times I} \alpha_{i j} e_{j}^{\prime} \otimes e_{i}$, and $v=\sum_{(i, j) \in I \times I} \beta_{i j} e_{j}^{\prime} \otimes e_{i}$, then $\lim _{i \in I} \alpha_{i j} e_{i}=0=\lim _{i \in I} \beta_{i j} e_{i}$ for each $j \in I$, and $\lim _{i \in I} \alpha_{i j} e_{i}=0=\lim _{i \in I} \beta_{i j} e_{i}$ for each $j \in I$. We have $u \circ v=\sum_{(i, j) \in I \times I}\left(\sum_{k \in I} \alpha_{i k} \beta_{k j}\right) e_{j}^{\prime} \otimes e_{i}$. Let $i \in I, \lim _{k \in I} \alpha_{i k} e_{k}^{\prime}=0$, that is, for each $\varepsilon>0$, there exists $J_{\varepsilon}(i)$ a finite subset of $I$ such that for each $k \notin J_{\varepsilon}(i)$, $\left\|\alpha_{i k} e_{k}^{\prime}\right\|<\varepsilon$. Hence

$$
\begin{aligned}
\left\|\left(\sum_{k \in I} \alpha_{i k} \beta_{k j}\right) e_{j}^{\prime}\right\| & =\left\|\sum_{k \in J_{\varepsilon}(i)}\left(\alpha_{i k} \beta_{k j}\right) e_{j}^{\prime}+\sum_{k \notin J_{\varepsilon}(i)}\left(\alpha_{i k} \beta_{k j}\right) e_{j}^{\prime}\right\| \\
& \leq \max \left(\max _{k \in J_{\varepsilon}(i)}\left\|\alpha_{i k} e_{k}^{\prime}\right\|\left\|e_{i}\right\|\left\|\beta_{k j} e_{k}\right\|\left\|e_{i}^{\prime}\right\|\left\|e_{j}^{\prime}\right\|, \sup _{k \notin J_{\varepsilon}(i)}\left\|\alpha_{i k} \beta_{k j} e_{j}^{\prime}\right\|\right) \\
& \leq \max \left(\|u\| \max _{k \in J_{\varepsilon}(i)}\left\|\beta_{k j} e_{j}^{\prime}\right\|\left\|e_{k}\right\|\left\|e_{i}^{\prime}\right\|, \varepsilon\|v\|\left\|e_{i}^{\prime}\right\|\right) .
\end{aligned}
$$

Since $\lim _{j \in I}\left\|\beta_{k j} e_{j}\right\|=0$ for each $k \in J_{\varepsilon}(i)$, we have $\lim _{j \in I}\left\|\left(\sum_{k \in I} \alpha_{i k} \beta_{k j}\right) e_{j}^{\prime}\right\|=0$ for each $i \in I$, therefore $u \circ v \in \mathscr{L}_{0}(E)$. The identity map id being given by id $=\sum_{i \in I} e_{i}^{\prime} \otimes e_{i}$, we have $\alpha_{i i}=1$ and $\alpha_{i j}=0$ if $i \neq j$. Therefore $\lim _{i} \alpha_{i j} e_{i}=0$ for each $j \in I$, and $\lim _{j} \alpha_{i j} e_{j}^{\prime}=0$ for each $i \in I$. Hence id $\in \mathscr{L}_{0}(E)$. Let $u=\sum_{(i, j) \in I \times I} \alpha_{i j} e_{j}^{\prime} \otimes e_{i}$ be in the closure of $\mathscr{L}_{0}(E)$. For all $\varepsilon>0$, there exists $u_{\varepsilon}=\sum_{(i, j) \in I \times I} \alpha_{i j}(\varepsilon) e_{j}^{\prime} \otimes e_{i} \in \mathscr{L}_{0}(E)$ such that $\left\|u-u_{\varepsilon}\right\|=\sup _{i, j}\left|\alpha_{i j}-\alpha_{i j}(\varepsilon)\right|\left\|e_{j}^{\prime}\right\|\left\|e_{i}\right\|<\varepsilon$. Hence for all $i, j \in I$, we have $\left|\alpha_{i j}\right|\left\|e_{j}^{\prime}\right\|\left\|e_{i}\right\| \leq \max \left(\varepsilon,\left|\alpha_{i j}(\varepsilon)\right|\left\|e_{j}^{\prime}\right\|\left\|e_{i}\right\|\right)$. We obtain $\lim _{i}\left\|\alpha_{i j} e_{i}\right\|=0$ for each $j \in I$ and $\lim _{j}\left\|\alpha_{i j} e_{j}^{\prime}\right\|=0$ for each $i \in I$. Therefore $u \in \mathscr{L}_{0}(E)$ and $\mathscr{L}_{0}(E)$ is closed.

2.2. Suppose that the orthogonal basis is orthonormal, that is, $\left\|e_{j}\right\|=1$ for each $j \in I$. Then $u=\sum_{(i, j) \in I \times I} \alpha_{i j} e_{j}^{\prime} \otimes e_{i} \in \mathscr{L}_{0}(E)$, if and only if $\lim _{i} \alpha_{i j}=0$ for each $j \in I$ and $\lim _{j} \alpha_{i j}=0$ for each $i \in I$. Setting for $u=\sum_{(i, j) \in I \times I} \alpha_{i j} e_{j}^{\prime} \otimes e_{i} \in \mathscr{L}_{0}(E), u^{*}=$ $\sum_{(i, j) \in I \times I} \alpha_{j i} e_{j}^{\prime} \otimes e_{i}$, we see that $u^{*} \in \mathscr{L}_{0}(E)$, called the adjoint of $u$. We verify easily the following proposition.

Proposition 2.2. An element $u \in \mathscr{L}(E)$ has an adjoint $u^{*}$ if and only if $u \in \mathscr{L}_{0}(E)$. Let $u, v \in \mathscr{L}_{0}(E), \lambda \in \mathbb{K}$. Then $(u+\lambda v)^{*}=u^{*}+\lambda v^{*} ;(u \circ v)^{*}=v^{*} \circ u^{*} ; u^{* *}=u$. Moreover, $\left\|u^{*}\right\|=\|u\|$. 
As usual, we say that $u \in \mathscr{L}_{0}(E)$ is normal (resp., unitary) if $u \circ u^{*}=u^{*} \circ u$ (resp., $u \circ u^{*}=\mathrm{id}=u^{*} \circ u$ ). And $u$ is selfadjoint if $u=u^{*}$, this is equivalent here to say that the matrix of $u$ is symmetric.

Note 2.3. (i) We have $\|u\|=\left\|u^{*}\right\|$. However, in general $\left\|u \circ u^{*}\right\| \neq\|u\|^{2}$. For example, if $I$ is the set of positive integers, and $E$ with orthogonal base $\left(e_{n}: n \geq 1\right)$, let $a, b \in \mathbb{K}$. The operator $u$ defined by $u\left(e_{1}\right)=a e_{1}+b e_{2}, u\left(e_{2}\right)=b e_{1}-a e_{2}, u\left(e_{3}\right)=c e_{3}$, and $u\left(e_{n}\right)=0$ for $n \geq 4$. We see that $u$ is selfadjoint. If $i=\sqrt{-1} \in \mathbb{K}$; then taking $b=i a$ and $|c|<|a|$, we see that $\left\|u^{2}\right\|=|c|^{2}<|a|^{2}=\|u\|^{2}$.

(ii) It should be interesting to characterize the elements of $\mathscr{L}_{0}(E)$ that are normal, unitary. Considering, whenever the base of $E$ is orthonormal, the bilinear form $f$ on $E$ defined by $f(x, y)=\sum_{i \in I} x_{i} y_{i}$, we obtain that the above definition of an adjoint $u^{*}$ of an element $u \in \mathscr{L}_{0}(E)$ is equivalent to say that $f(u(x), y)=f\left(x, u^{*}(y)\right)$ for each $x$ and $y \in E$. In fact, here the adjoint of an operator is its transposition. This example is related to ultrametric Hilbert spaces.

3. Ultrametric Hilbert spaces. For the so-called ultrametric Hilbert spaces we can also define the adjoint of an operator with respect to an appropriate bilinear symmetric form.

3.1. Remark and definition. Ochsenius and Schikhof write in [24] "as a slogan: There are no $p$-adic Hilbert spaces." Nevertheless we will give a definition of $p$-adic Hilbert spaces (cf. [20, 21] for some fields with infinite rank valuation). Let $\omega=\left(\omega_{i}\right)_{i \geq 0}$ be a sequence of nonzero elements of $\mathbb{K}$. We consider the free Banach space $E_{\omega}=$ $c_{0}\left(\mathbb{N}, \mathbb{K},\left(\left|\omega_{i}\right|^{1 / 2}\right)_{i \geq 0}\right)=\left\{x: x=\left(x_{i}\right)_{i \geq 0} \subset \mathbb{K} ; \lim _{i \rightarrow+\infty}\left|x_{i}\right|\left|\omega_{i}\right|^{1 / 2}=0\right\}$. Then $x=\left(x_{i}\right)_{i \geq 0}$ $\in E_{\omega} \leftrightarrow \lim _{i \rightarrow+\infty} x_{i}^{2} \omega_{i}=0$. Setting $e_{i}=\left(\delta_{i, j}\right)_{j \geq 0}$ (Kronecker symbol), we have that $\left(e_{i}: i \geq 0\right)$ is an orthogonal base of $E_{\omega}$ : for all $x \in E_{\omega}, x=\sum_{i \geq 0} x_{i} e_{i}$ and $\|x\|=$ $\sup _{i \geq 0}\left|x_{i}\right|\left\|e_{i}\right\|=\sup _{i \geq 0}\left|x_{i}\right|\left|\omega_{i}\right|^{1 / 2}$, in particular, $\left\|e_{i}\right\|=\left|\omega_{i}\right|^{1 / 2}$ for each $i \geq 0$. Let $f_{\omega}: E_{\omega} \times E_{\omega} \rightarrow \mathbb{K}$ be defined by $f_{\omega}(x, y)=\sum_{i \geq 0} \omega_{i} x_{i} y_{i}$. It is readily seen that $f_{\omega}$ is a bilinear symmetric form on $E_{\omega}$, with $\left|f_{\omega}(x, y)\right| \leq\|x\|\|y\|$, that is, the bilinear form $f_{\omega}$ is continuous. Moreover, $f_{\omega}$ is nondegenerate, that is, $f_{\omega}(x, y)=0$ for each $y \in E_{\omega} \Rightarrow x=0$. Furthermore, $f_{\omega}(x, x)=\sum_{i \geq 0} \omega_{i} x_{i}^{2}$ and $f_{\omega}\left(e_{i}, e_{j}\right)=\omega_{i} \delta_{i, j}$ for $i$ and $j \geq 0$. The space $E_{\omega}$ is called a $p$-adic Hilbert space.

Note 3.1. (i) It may happen that $\left|f_{\omega}(x, x)\right|<\|x\|^{2}$ for some $x \in E_{\omega}$ and even worse, $E_{\omega}$ contains isotropic elements $x \neq 0$, that is, $f_{\omega}(x, x)=0$.

(ii) Let $V$ be a subspace of $E_{\omega}$ and $V^{\perp}=\left\{x \in E_{\omega}: f_{\omega}(x, y)=0\right.$, for all $\left.y \in V\right\}$. The fundamental property on subspaces of the classical Hilbert space $H: V=V^{\perp \perp} \Rightarrow$ $V \oplus V^{\perp}=H$ fails to be true in the $p$-adic case. This explains the claim of Ochsenius and Schikhof.

REMARK 3.2. A free Banach space $E$ with an orthogonal base $\left(e_{i}: i \geq 0\right)$ can be given a structure of a $p$-adic Hilbert space if and only if there exists $\left(\omega_{i}: i \geq 0\right) \subset \mathbb{K}$ such that $\left\|e_{i}\right\|=\left|\omega_{i}\right|^{1 / 2}$ for each $i \geq 0$. Furthermore, if $\mathbb{K}$ contains a square of any of its element, then any $p$-adic Hilbert is isomorphic, in a natural way, to the space $c_{0}(\mathbb{N}, \mathbb{K})$. 
Note 3.3. Let $u, v \in \mathscr{L}\left(E_{\omega}\right)$; we have $u=\sum_{i, j} \alpha_{i j} e_{j}^{\prime} \otimes e_{i}$ and $v=\sum_{i, j} \beta_{i j} e_{j}^{\prime} \otimes e_{i}$ with $\lim _{i \rightarrow+\infty}\left|\alpha_{i j}\right|\left|\omega_{i}\right|^{1 / 2}=0=\lim _{i \rightarrow+\infty}\left|\beta_{i j}\right|\left|\omega_{i}\right|^{1 / 2}$ for each $j \geq 0$. Furthermore, the norm of $u \in \mathscr{L}\left(E_{\omega}\right)$ is given by

$$
\|u\|=\sup _{i, j} \frac{\left|\omega_{i}\right|^{1 / 2}\left|\alpha_{i j}\right|}{\left|\omega_{j}\right|^{1 / 2}}
$$

The operator $v$ is said to be an adjoint of $u$ with respect to $f_{\omega} \Leftrightarrow f_{\omega}(u(x), y)=$ $f_{\omega}(x, v(y))$, for all $x, y \in E_{\omega}$. Since $f_{\omega}$ is symmetric, $u$ is an adjoint of $v$.

Since $f_{\omega}$ is nondegenerate, if an operator $u$ has an adjoint, this adjoint is unique and is denoted by $u^{*}$. Since $\left(e_{i}: i \geq 0\right)$ is an orthogonal base of $E_{\omega}$, we have that $v$ is an adjoint of $u$ if and only if $f_{\omega}\left(\left(u\left(e_{i}\right), e_{j}\right)=f_{\omega}\left(e_{i}, v\left(e_{j}\right)\right)\right)$ for each $i$ and $j \geq 0$. That is, $f_{\omega}\left(\sum_{k \geq 0} \alpha_{k i} e_{k}, e_{j}\right)=\alpha_{j i} \omega_{j}=f_{\omega}\left(e_{i}, \sum_{k \geq 0} \beta_{k j} e_{k}\right)=\beta_{i j} \omega_{i}$, for all $i, j \geq 0 \Leftrightarrow$ $\beta_{i j}=\omega_{i}^{-1} \omega_{j} \alpha_{j i}$, for all $i, j \geq 0$. Furthermore, we must have $\lim _{i \rightarrow+\infty}\left|\beta_{i j}\right|\left|\omega_{i}\right|^{1 / 2}=0$ for each $j \geq 0$, that is,

$$
\lim _{i \rightarrow+\infty}\left|\omega_{i}\right|^{1 / 2}\left|\omega_{j}^{-1}\right|\left|\omega_{j}\right|\left|\alpha_{j i}\right|=\left|\omega_{j}\right| \lim _{i \rightarrow+\infty}\left|\omega_{i}\right|^{-1 / 2}\left|\alpha_{j i}\right|=0, \quad \forall j \geq 0
$$

Hence $\lim _{i \rightarrow+\infty}\left|\omega_{i}\right|^{-1 / 2}\left|\alpha_{j i}\right|=0$ for each $j \geq 0$. We have proved the following theorem.

THEOREM 3.4. Let $\left(\omega_{i}\right)_{i \geq 0} \subset \mathbb{K}^{*}$ and $E_{\omega}=c_{0}\left(\mathbb{N}, \mathbb{K},\left(\left|\omega_{i}\right|^{1 / 2}\right)_{i \geq 0}\right)$ be the $p$-adic Hilbert space associated with $\omega$. Let $u=\sum_{i, j} \alpha_{i j} e^{\prime}{ }_{j} \otimes e_{i} \in \mathscr{L}\left(E_{\omega}\right)$. Then $u$ has an adjoint $v=u^{*} \in \mathscr{L}\left(E_{\omega}\right)$ if and only if $\lim _{j \rightarrow+\infty}\left|\omega_{j}\right|^{-1 / 2}\left|\alpha_{i j}\right|=0$ for each $i \geq 0$. In this condition, $u^{*}=\sum_{i, j} \omega_{i}^{-1} \omega_{j} \alpha_{j i} e^{\prime}{ }_{j} \otimes e_{i}$.

It follows from this theorem that not any continuous linear operator of $E_{\omega}$ has an adjoint: it is another difference with classical Hilbert spaces. Let $\mathscr{L}_{0}\left(E_{\omega}\right)=\{u: u=$ $\left.\sum_{i \geq 0} \sum_{j \geq 0} \alpha_{i j} e_{j}^{\prime} \otimes e_{i} \in \mathscr{L}\left(E_{\omega}\right) ; \lim _{j \rightarrow+\infty}\left|\omega_{j}\right|^{-1 / 2}\left|\alpha_{i j}\right|=0, \forall i \geq 0\right\}$. We remember that $u=\sum_{i, j} \alpha_{i j} e_{j}^{\prime} \otimes e_{i} \in \mathscr{L}\left(E_{\omega}\right)$ is equivalent to $\lim _{i \rightarrow+\infty}\left|\omega_{i}\right|^{1 / 2}\left|\alpha_{i j}\right|=0$ for each $j \geq 0$. It is readily seen, as in Theorem 2.1 , that $\mathscr{L}_{0}\left(E_{\omega}\right)$ is a closed unitary subalgebra of $\mathscr{L}\left(E_{\omega}\right)$.

COROLlary 3.5. An element $u \in \mathscr{L}\left(E_{\omega}\right)$ has an adjoint $u^{*}$ if and only if $u \in \mathscr{L}_{0}\left(E_{\omega}\right)$. Let $u, v \in \mathscr{L}_{0}\left(E_{\omega}\right), \lambda \in \mathbb{K}$. Then $(u+\lambda v)^{*}=u^{*}+\lambda v^{*} ;(u \circ v)^{*}=v^{*} \circ u^{*} ; u^{* *}=u$. Moreover, $\left\|u^{*}\right\|=\|u\|$.

Proof. We only prove that $\left\|u^{*}\right\|=\|u\|$. Since for $u=\sum_{i, j} \alpha_{i j} e_{j}^{\prime} \otimes e_{i} \in \mathscr{L}_{0}\left(E_{\omega}\right)$, we have $\|u\|=\sup _{i, j}\left(\left|\omega_{i}\right|^{1 / 2}\left|\alpha_{i j}\right| /\left|\omega_{j}\right|^{1 / 2}\right)$ and $u^{*}=\sum_{i, j} \omega_{j} \omega_{i}^{-1} \alpha_{j i} e_{j}^{\prime} \otimes e_{i}$, we obtain

$$
\left\|u^{*}\right\|=\sup _{i, j} \frac{\left|\omega_{i}\right|^{1 / 2}}{\left|\omega_{j}\right|^{1 / 2}}\left|\omega_{j}\right|\left|\omega_{i}^{-1}\right|\left|\alpha_{i j}\right|=\sup _{i, j} \frac{\left|\omega_{j}\right|^{1 / 2}}{\left|\omega_{i}\right|^{1 / 2}}\left|\alpha_{j i}\right|=\|u\| .
$$

REMARK 3.6. (i) $u=\sum_{i, j} \alpha_{i j} e_{j}^{\prime} \otimes e_{i} \in \mathscr{L}_{0}\left(E_{\omega}\right)$ is selfadjoint, that is, $u=u^{*}$ if and only if $\alpha_{j i}=\omega_{i} \omega_{j}^{-1} \alpha_{i j}$, for each $i \geq 0$ and each $j \geq 0$.

(ii) Examples of selfadjoint operators on ultrametric Hilbert spaces and study of their spectrum are given in [1, 2, 6, 22]. 


\section{Closed subalgebras generated by projectors}

4.1. Let $J$ be a subset of $I$ and $E$ be a free Banach space with orthogonal basis $\left(e_{j}: j \in I\right)$. The linear operator $p_{J}=\sum_{i \in J} e_{i}^{\prime} \otimes e_{i}$ of $E$ belongs to $\mathscr{L}_{0}(E)$. Let $\mathscr{D}=\{u: u=$ $\left.\sum_{i \in I} \lambda_{i} e_{i}^{\prime} \otimes e_{i} \in \mathscr{L}_{0}(E) ; \sup _{i \in I}\left|\lambda_{i}\right|<+\infty\right\}$. It is clear that $\mathscr{D}$ is isometrically isomorphic to the algebra of bounded families $\ell^{\infty}(I, \mathbb{K})$. Let $\operatorname{Hom}_{\text {alg }}(\mathscr{D}, \mathbb{K})$ denotes the family of all algebra homomorphisms of $\mathscr{D}$ into $\mathbb{K}$. Consider the spectrum $\mathscr{L}(\mathscr{D})=\operatorname{Hom}_{\mathrm{alg}}(\mathscr{D}, \mathbb{K})$ in a topology inherited from the Tihonov topology of the product $\mathbb{K}^{\mathscr{D}}$ of copies of $\mathbb{K}$.

Proposition 4.1. (i) An element $u=\sum_{i \in I} \lambda_{i} e_{i}^{\prime} \otimes e_{i} \in \mathscr{D}$ is an idempotent if and only if there exists $J \subset I$ such that $u=p_{J}$.

(ii) The spectrum $\mathscr{L}(\mathscr{D})$ is homeomorphic to the subset of ultrafilters on $I: \Phi_{c}=\{\mathscr{U}$ : $\mathcal{U}$ is an ultrafilter on $I$, such that for all $u=\sum_{i \in I} \lambda_{i} e_{i}^{\prime} \otimes e_{i} \in \mathscr{D}$, the limit $\lim _{\mathcal{U}} \lambda_{i}$ exists in $\mathbb{K}\}$.

Proof. (i) Let $u=\sum_{i \in I} \lambda_{i} e_{i}^{\prime} \otimes e_{i}$; then $u \circ u=u$ if and only if $\sum_{i \in I} \lambda_{i}^{2} e_{i}^{\prime} \otimes e_{i}=$ $\sum_{i \in I} \lambda_{i} e_{i}^{\prime} \otimes e_{i}$, if and only if $\lambda_{i}^{2}=\lambda_{i}$ for each $i \in I$, if and only if $\lambda_{i}=0$ or $\lambda_{i}=1$. Setting $J=\left\{i: i \in I ; \lambda_{i}=1\right\}$, we have $u=p_{J}$.

(ii) Let $\chi$ be a character of $\mathscr{D}$, that is, an algebra homomorphism (necessarily continuous) of $\mathscr{D}$ into $\mathbb{K}$. For all $J, L \subset I$ we have $p_{J} \circ p_{L}=p_{J \cap L}$, hence $p_{J} \circ p_{J^{c}}=p_{\varnothing}=0$, where $J^{c}=I \backslash J$. Furthermore, $\chi\left(p_{J}\right)=\chi\left(p_{J}\right) \chi\left(p_{J}\right)$ implies that $\chi\left(p_{J}\right)=0$ or 1 . Let $U_{\chi}=\{J$ : $\left.J \subset I ; \chi\left(p_{J}\right)=1\right\}$. This family of subsets is an ultrafilter. Indeed, $\varnothing \notin \boldsymbol{U}_{\chi}$. If $J \subset L$ with $J \in u_{\chi}$, then $1=\chi\left(p_{J}\right)=\chi\left(p_{J \cap L}\right)=\chi\left(p_{J}\right) \chi\left(p_{L}\right)=\chi\left(p_{L}\right)$, hence $L \in U_{\chi}$. On the other hand, for $J \subset I$, we have $1_{E}=p_{J}+p_{J^{c}}$, and $1=\chi\left(1_{E}\right)=\chi\left(p_{J}\right)+\chi\left(p_{J^{c}}\right)$ with $\chi\left(p_{J}\right)=1$ or 0 and $\chi\left(p_{J^{c}}\right)=1$ or 0 . If $\chi\left(p_{J}\right)=1$, then $\chi\left(p_{J^{c}}\right)=0$, and if $\chi\left(p_{J^{c}}\right)=1$, we have $\chi\left(p_{J}\right)=$ 0 . Hence $J \in \mathcal{U}_{\chi}$ or $J^{c} \in \mathcal{U}_{\chi}$. Let $u=\sum_{i \in I} \lambda_{i} e_{i}^{\prime} \otimes e_{i} \in \mathscr{D}$. Put $\chi(u)=\lambda \in \mathbb{K}$; then for all $J \in u_{\chi}, \chi\left(\operatorname{up}_{J}\right)=\chi(u)=\lambda \chi\left(p_{J}\right)$. Therefore $\chi\left(\operatorname{up}_{J}-\lambda p_{J}\right)=0$, that is, up $\operatorname{up}_{J}-\lambda p_{J} \in \operatorname{ker} \chi$. Set $\phi_{U_{\chi}}(u)=\lim _{U_{X}}\left|\lambda_{i}\right|$. It is well known and readily seen that $\phi_{U_{\chi}}$ is a multiplicative semi-norm on $\mathscr{D}$ and that $\operatorname{ker} \phi_{\cup_{X}}=\left\{u: u \in \mathscr{D} ; \phi_{\cup_{X}}(u)=0\right\}$ is a maximal ideal of $\mathscr{D}$, since $\mathscr{D}$ is isomorphic to $\ell^{\infty}(I, \mathbb{K})$. On the other hand $\left|\chi\left(u_{J}\right)\right| \leq\left\|\operatorname{up}_{J}\right\|=\sup _{i \in J}\left|\lambda_{i}\right|$ for each $J \in u_{\chi}$. It follows that $|\chi(u)|=\left|\chi\left(\operatorname{up}_{J}\right)\right| \leq \inf _{J \in U_{\chi}} \sup _{i \in J}\left|\lambda_{i}\right|=\phi_{u_{\chi}}(u)$. Hence, $\operatorname{ker} \phi_{U_{\chi}} \subset \operatorname{ker} \chi$ and $\operatorname{ker} \phi_{U_{X}}=\operatorname{ker} \chi$. Let $J \in U_{\chi}$, we deduce from $\left(\operatorname{up}_{J}-\lambda p_{J}\right) \in \operatorname{ker} \chi=$ $\operatorname{ker} \phi_{\cup_{\chi}}$, that $0=\phi_{\cup_{X}}\left(\operatorname{up}_{J}-\lambda p_{J}\right)=\lim _{\cup_{X}}\left|\lambda_{i}-\lambda\right|$. It follows that $\lim _{\cup_{\chi}} \lambda_{i}=\lambda$ exists in $\mathbb{K}$. Moreover, $\chi(u)=\lambda=\lim _{\cup_{\chi}}\left|\lambda_{i}\right|$, and we see that $\chi=\chi \cup_{\chi}$. Reciprocally, if $u$ is an ultrafilter on $I$ such that for all $u=\sum_{i \in I} \lambda_{i} e_{i}^{\prime} \otimes e_{i} \in \mathscr{D}, \lim _{\imath} \lambda_{i}$ exists in $\mathbb{K}$; then setting $\chi_{\varkappa}(u)=\lim _{\varkappa} \lambda_{i}$, it is readily seen that $\chi_{\varkappa}$ is a character of $\mathscr{D}$. Moreover, for all $J \in U$, $\chi_{\varkappa}\left(p_{J}\right)=\lim _{U} 1=1$, that is, $J \in \mathcal{u}_{\chi_{\varkappa}}$ and $U=u_{\chi_{\varkappa}}$. The proposition is proved if we consider on $\mathscr{X}(\mathscr{D})$ the weak*-topology and on $\Phi_{c}$ the topology induced by the natural topology on the space of ultrafilters, which is the weakest topology on $\Phi_{c}$ relative to which the mapping $\lim : \Phi_{c} \rightarrow \mathbb{K}$ is continuous.

REMARK 4.2. (i) If $\mathbb{K}$ is locally compact, then for any bounded family $\left(\lambda_{i}\right)_{i \in I} \subset \mathbb{K}$, the limit $\lim _{\vartheta} \lambda_{i}$ exists in $\mathbb{K}$. Therefore, $\Phi_{c}$ is equal to the entire set of all ultrafilters on $I$ and $\mathscr{X}(\mathscr{D})$ is compact, homeomorphic to the Stone-Čech compactification $\beta(I)$ of the discrete topological space $I$.

(ii) If $\mathbb{K}$ is not spherically complete and $I$ is a small set, that is, the cardinal of $I$ is nonmeasurable, it is well known that the continuous dual of $\ell^{\infty}(I, \mathbb{K})$ is equal to the 
space $c_{0}(I, \mathbb{K})$ of the families converging to zero (cf. [28, Theorem 4.21]). Then, we can prove that $\mathscr{L}(\mathscr{D})$ is homeomorphic with $I$.

Note 4.3. For $\mathbb{K}$ spherically complete, not locally compact, it is interesting to find explicit conditions on an ultrafilter $U$ in such a way that $\lim _{\imath} \lambda_{i}$ exists for any bounded family $\left(\lambda_{i}: i \in I\right) \subset \mathbb{K}$. We can try to use Banach limits, that is, continuous linear forms on $\ell^{\infty}(I, \mathbb{K})$ that extend the usual continuous linear form of the limit operation defined on the subspace $c_{v}(I, \mathbb{K})$ of convergent families.

Let $\left(J_{v}: v \in \Lambda\right)$ be a family of subsets of $I$, such that $J_{v} \cap J_{\mu}=\varnothing$ for $v \neq \mu$. Putting $p_{v}=\sum_{i \in J_{v}} e_{i}^{\prime} \otimes e_{i}$, we obtain $p_{v} \circ p_{\mu}=\delta_{v, \mu} p_{v}$, for $v \neq \mu$. Hence the subalgebra with the unity $\mathscr{B}$ of $\mathscr{L}_{0}(E)$, generated by $\left(p_{v}: v \in \Lambda\right)$ is equal to $\mathbb{K} \cdot \operatorname{id} \oplus\left(\oplus_{v \in \Lambda} \mathbb{K} \cdot p_{v}\right)$. Indeed if $u=\alpha_{0}$ id $+u_{1}$ and $v=\beta_{0}$ id $+v_{1}$ with $u_{1}=\sum_{v \in \Lambda} \alpha_{v} p_{v}$ and $v_{1}=\sum_{v \in \Lambda} \beta_{v} p_{v}$ (finite sums), we have $u \circ v=\alpha_{0} \beta_{0} \mathrm{id}+\alpha_{0} v_{1}+\beta_{0} u_{1}+u_{1} \circ v_{1}=\alpha_{0} \beta_{0} \mathrm{id}+\sum_{v \in \Lambda}\left(\alpha_{0} \beta_{v}+\right.$ $\left.\alpha_{v} \beta_{0}+\alpha_{v} \beta_{v}\right) p_{v} \in \mathscr{B}$. On the other hand, since $u=\alpha_{0}$ id $+\sum_{v \in \Lambda} p_{v}$ with $\Gamma=\{v: v \in$ $\left.\Lambda ; \alpha_{v} \neq 0\right\}$ finite and $I=\left(\bigcup_{v \in \Gamma} J_{v}\right) \bigcup\left(\bigcap_{v \in \Gamma} J_{v}{ }^{c}\right)$ (a partition), we have $u=\alpha_{0} \sum_{i \in I} e_{i}^{\prime} \otimes$ $e_{i}+\sum_{v \in \Gamma} \alpha_{v} \sum_{i \in J_{v}} e_{i}^{\prime} \otimes e_{i}=\alpha_{0} \sum_{i \in \cap_{v} \in \Gamma J_{v}} e_{i}^{\prime} \otimes e_{i}+\sum_{v \in \Gamma} \sum_{i \in J_{v}}\left(\alpha_{0}+\alpha_{v}\right) e_{i}^{\prime} \otimes e_{i}$. Hence $\|u\|=\max \left(\left|\alpha_{0}\right|, \max _{v}\left|\alpha_{0}+\alpha_{v}\right|\right)$.

LEMMA 4.4. Let $u=\alpha_{0} \mathrm{id}+\sum_{v \in \Lambda} \alpha_{v} p_{v} \in \mathscr{B}$ and $\Lambda_{0}=\Lambda \cup\{0\}$. Then $\|u\|=$ $\max _{v \in \Lambda_{0}}\left|\alpha_{v}\right|$. That is, $\{\mathrm{id}\} \cup\left\{p_{v}: v \in \Lambda\right\}$ is an orthonormal family in $\mathscr{L}_{0}(E)$.

Proof. Since

$$
\begin{gathered}
\|u\|=\max \left(\left|\alpha_{0}\right|, \max _{v \in \Lambda}\left|\alpha_{0}+\alpha_{v}\right|\right), \\
\max _{v}\left|\alpha_{0}+\alpha_{v}\right| \leq \max \left(\left|\alpha_{0}\right|, \max _{v \in \Lambda}\left|\alpha_{v}\right|\right) .
\end{gathered}
$$

We have $\|u\| \leq \max _{v \in \Lambda_{0}}\left|\alpha_{v}\right|$. Moreover, $\left|\alpha_{0}\right| \leq\|u\|$. Hence for $v \in \Lambda$, we have $\left|\alpha_{v}\right|=$ $\left|\alpha_{v}+\alpha_{0}-\alpha_{0}\right| \leq \max \left(\left|\alpha_{v}+\alpha_{0}\right|,\left|\alpha_{0}\right|\right) \leq\|u\|$. It follows that $\max _{v \in \Lambda_{0}}\left|\alpha_{v}\right| \leq\|u\|$, and Lemma 4.4 is proved.

LEMMA 4.5. Assume that $\left(e_{i}: i \in I\right)$ is an orthonormal basis of $E$ or $E$ is an ultrametric Hilbert space. Then any $u \in \mathscr{B}$ is selfadjoint, that is, $u^{*}=u$, and $\left\|u^{2}\right\|=\|u\|^{2}$.

Proof. That any element of $\mathscr{R}$ is selfadjoint is easy to verify. Let $u=\alpha_{0}$ id + $\sum_{v \in \Lambda} \alpha_{v} p_{v} \in \mathscr{B}$, we have $u^{2}=\alpha_{0}^{2} \mathrm{id}+\sum_{v \in \Lambda}\left(2 \alpha_{0} \alpha_{v}+\alpha_{v}^{2}\right) p_{v} \in \mathscr{B}$. Hence $\left\|u^{2}\right\|=$ $\max \left(\left|\alpha_{0}\right|^{2}, \max _{v \in \Lambda}\left|\alpha_{0}{ }^{2}+2 \alpha_{0} \alpha_{v}+\alpha_{v}{ }^{2}\right|\right)=\left(\max \left(\left|\alpha_{0}\right|, \max _{v \in \Lambda}\left|\alpha_{0}+\alpha_{v}\right|\right)\right)^{2}=\|u\|^{2}$.

Note 4.6. In fact, Lemma 4.5 is true for $u \in \mathscr{D}$. Let $E$ be a free Banach space with orthogonal basis $\left(e_{i}: i \in I\right)$. Fix $\pi \in \mathbb{K}$ such that $0<|\pi|<1$. There exists for any $i \in I$ an integer $n_{i} \in \mathbb{Z}$ such that $|\pi|^{n_{i}+1}<\left\|e_{i}\right\| \leq|\pi|^{n_{i}}$. For $x=\sum_{i \in I} x_{i} e_{i}$, we have $\lim _{i \in I} x_{i} \pi^{n_{i}}=0$. Hence we define on $E$ a norm by setting $\|x\|_{\pi}=\sup _{i \in I}\left|x_{i}\right||\pi|^{n_{i}}$; this norm is equivalent to \|\| with $|\pi|\|x\|_{\pi} \leq\|x\| \leq\|x\|_{\pi}$. Furthermore, setting $x=$ $\sum_{i \in I} x_{i} e_{i}$ and $y=\sum_{i \in I} y_{i} e_{i} \in E, f_{\pi}(x, y)=\sum_{i \in I} \pi^{2 n_{i}} x_{i} y_{i}$, we have a continuous, non degenerated, bilinear form on $E$ such that $\left|f_{\pi}(x, y)\right| \leq\|x\|_{\pi}\|y\|_{\pi} \leq|\pi|^{-2}\|x\|\|y\|$. Therefore, we obtain on $E$, a structure of ultrametric Hilbert space $E_{\pi}=\left(E,\|\|_{\pi}, f_{\pi}\right)$. Since the norms \|\| and \|\|$_{\pi}$ are equivalent, $\mathscr{L}(E)=\mathscr{L}\left(E_{\pi}\right)$ and $\mathscr{L}_{0}(E)=\mathscr{L}_{0}\left(E_{\pi}\right)$. 
The norms on $\mathscr{L}(E)$ induced by \|\| and \|\|$_{\pi}$ are equivalent with $|\pi|\|u\|_{\pi} \leq\|u\| \leq$ $|\pi|^{-1}\|u\|_{\pi}$. As in Note 3.3, we define the adjoint $u^{*}$ of $u \in \mathscr{L}(E)$ with respect to $f_{\pi}$. We obtain the results stated in Theorem 3.4, that is, $u$ admits an adjoint with respect to $f_{\pi}$ if and only if $u \in \mathscr{L}_{0}(E)$. Furthermore, if $u=\sum_{i, j} \alpha_{i j} e^{\prime}{ }_{j} \otimes e_{i} \in \mathscr{L}_{0}(E)$, then $u^{*}=\sum_{i, j} \pi^{n_{j}-n_{i}} \alpha_{j i} e^{\prime}{ }_{j} \otimes e_{i}$, and $u$ is selfadjoint, that is, $u^{*}=u$ if and only if $\pi^{n_{i}} \alpha_{i j}=\pi^{n_{j}} \alpha_{j i}$, for all $i, j \in I$.

Note 4.7. Let $\pi^{\prime}$ be another element of $\mathbb{K}$ such that $0<\left|\pi^{\prime}\right|<1$. Also let $\left(m_{i}: i \in\right.$ I) $\subset \mathbb{Z}$ be defined by $\left|\pi^{\prime}\right|^{m_{i}+1}<\left\|e_{i}\right\| \leq\left|\pi^{\prime}\right|^{m_{i}}$. Then the adjoint $u^{\dagger}=\sum_{i, j} \pi^{\prime m_{j}-m_{i}}$ $\alpha_{j i} e^{\prime}{ }_{j} \otimes e_{i}$ of $u$ with respect to $f_{\pi^{\prime}}$ coincides with $u^{*}$ if and only if $\pi^{n_{j}-n_{i}} \alpha_{j i}=$ $\pi^{\prime m_{j}-m_{i}} \alpha_{j i}$, for each $i$ and $j \in I$. If this is true for all $u \in \mathscr{L}_{0}(E)$, we have $\pi^{n_{j}-n_{i}}=$ $\pi^{\prime m_{j}-m_{i}}$, for $i, j \in I$. Hence, $\log |\pi| /|\log | \pi^{\prime} \mid=\left(m_{j}-m_{i}\right) /\left(n_{j}-n_{i}\right)=m / n>0$ and the sets $\left(m_{j}-m_{i}\right)_{i \neq j}$ and $\left(n_{j}-n_{i}\right)_{i \neq j}$ must be finite.

If $J$ is a subset of $I$, the projector $p_{J}=\sum_{i \in J} e_{i}^{\prime} \otimes e_{i}$ is selfadjoint with respect to any bilinear symmetric form $f_{\pi}$ and $\left\|p_{J}\right\|=1=\left\|p_{J}\right\|_{\pi}$.

LEMmA 4.8. Let $E$ be a free Banach space with orthogonal basis $\left(e_{i}: i \in I\right)$. Defining an adjoint of a continuous operator with respect to $f_{\pi}$, then any $u \in \mathscr{B}$ (resp., $\mathscr{D}$ ) is selfadjoint and $\left\|u^{2}\right\|=\|u\|^{2}$.

Proof. It is the same as in Lemma 4.5. Since for any $u=\alpha_{0} \mathrm{id}+\sum_{v \in \Lambda} \alpha_{v} p_{v} \in \mathscr{B}$ we have $\|u\|=\max _{v \in \Lambda_{0}}\left|\alpha_{v}\right|$, that is, $\left\{\mathrm{id}, p_{v}: v \in \Lambda\right\}$ is an orthonormal family in $\mathscr{L}_{0}(E)$, we see that the closure $\mathscr{A}=\overline{\mathscr{B}}$ of $\mathscr{B}$ is the subspace of $\mathscr{L}_{0}(E)$ of all elements $u$ which can be written in the unique form of summable families $u=\alpha_{0}$ id $+\sum_{v \in \Lambda} \alpha_{v} p_{v}$ with $\alpha_{0}, \alpha_{v} \in \mathbb{K}$ and $\lim _{v} \alpha_{v}=0$. It is readily seen that $\mathscr{A}$ is a closed unitary subalgebra of $\mathscr{L}_{0}(E)$, contained in $\mathscr{D}$, such that any element $u$ of $\mathscr{A}$ is selfadjoint. Moreover for the pointwise convergence, $u=\alpha_{0} \sum_{i \in \cap J_{v}^{c}} e_{i}^{\prime} \otimes e_{i}+\sum_{v \in \Lambda} \sum_{i \in J_{v}}\left(\alpha_{0}+\alpha_{v}\right) e_{i}^{\prime} \otimes$ $e_{i}$. Hence, if $\bigcap_{v \in \Lambda} J_{v}^{c}=\varnothing$, then $u=\sum_{v \in \Lambda} \sum_{i \in J_{v}}\left(\alpha_{0}+\alpha_{v}\right) e_{i}^{\prime} \otimes e_{i}$ and id $=\sum_{v \in \Lambda} p_{v}$.

EXAMPLE 4.9. If $\Lambda=I$ and $J_{i}=\{i\}$ for each $i \in I$, we have $\mathscr{A}=\left\{\alpha_{0}\right.$ id $+\sum_{i \in I} \alpha_{i} e_{i}^{\prime} \otimes e_{i}$ : $\left.\alpha_{i} \in \mathbb{K}, \lim _{i \in I} \alpha_{i}=0\right\}$. As an element of $\mathscr{D}$ any $u \in \mathscr{A}$ is in the form $u=\sum_{i \in I} a_{i} e_{i}^{\prime} \otimes e_{i}$ with $\lim _{i \in I} a_{i}=\alpha_{0}$ exists in $\mathbb{K}$.

Proposition 4.10. (i) Any element $u$ of the Banach algebra A with the unit element id is selfadjoint with respect to any bilinear symmetric form $f_{\pi}$ and $\left\|u^{2}\right\|=\|u\|^{2}$.

(ii) The spectrum $\mathscr{L}(\mathscr{A})=\operatorname{Hom}_{\mathrm{alg}}(\mathscr{A}, \mathbb{K})$ of $\mathscr{A}$, equipped with the weak ${ }^{*}$-topology, is homeomorphic to the Alexandroff compactification of the discrete space $\Lambda$.

Proof. The first part is an easy consequence of Lemma 4.8. Let $\chi \in \mathscr{X}(\mathscr{A})$, then $\chi$ is a continuous linear form with norm $\|\chi\|=1$. Furthermore, $\chi($ id $)=1$ and $\chi\left(p_{\nu} p_{\mu}\right)=$ $\chi\left(p_{v}\right) \chi\left(p_{\mu}\right)=\delta_{v, \mu} \chi\left(p_{\nu}\right)$, for $\nu, \mu \in \Lambda$. It follows that for any $v \in \Lambda, \chi\left(p_{v}\right)=1$ or $\chi\left(p_{\nu}\right)=0$. Hence (a) there exists $\nu \in \Lambda$ such that $\chi\left(p_{\nu}\right)=1$ and $\chi\left(p_{\mu}\right)=0$ for $\mu \neq \nu$, or (b) $\chi\left(p_{v}\right)=0$ for all $v \in \Lambda$. In case (a), we put $\chi=\chi_{v}$ and in case (b), $\chi=\chi_{0}$. We verify that for $u=\alpha_{0} \mathrm{id}+\sum_{v \in \Lambda} \alpha_{v} p_{v} \in \mathcal{A}$, we have $\chi_{0}(u)=\alpha_{0}$ and $\chi_{v}(u)=\alpha_{0}+\alpha_{v}$, $v \in \Lambda$. It follows that $\mathscr{L}(\mathscr{A})=\left\{\chi_{0}, \chi_{v}: v \in \Lambda\right\}$ and $\mathscr{L}(\mathscr{A})$ is in a bijective correspondence with the set $\Lambda_{0}=\Lambda \cup\{0\}$. Let $W\left(\chi ; \varepsilon, u_{1}, \ldots, u_{n}\right)=\left\{\eta: \eta \in \mathscr{X}(\mathscr{D}) ;\left|\chi\left(u_{j}\right)-\eta\left(u_{j}\right)\right|<\varepsilon\right.$, 
$\left.u_{j} \in \mathscr{A}, 1 \leq j \leq n\right\}$ be a fundamental neighborhood of $\chi \in \mathscr{L}(\mathscr{A})$ for the weak*topology. Since for $u_{j}=\alpha_{0 j}$ id $+\sum_{\mu \in \Lambda} \alpha_{\mu j} p_{\mu} \in \mathscr{A}, \lim _{\mu \in \Lambda} \alpha_{\mu j}=0$, there exists a finite subset $\Gamma_{\varepsilon}$ of $\Lambda$, such that for any $\mu \notin \Gamma_{\varepsilon},\left|\alpha_{\mu j}\right|<\varepsilon$ for each $1 \leq j \leq n$. If $\chi=\chi_{v}, v \in \Lambda$, we have for $1 \leq j \leq n, \mu \in \Lambda, \chi_{v}\left(u_{j}\right)-\chi_{\mu}\left(u_{j}\right)=\alpha_{v j}-\alpha_{\mu j}$. Choosing $\left(u_{j}: 1 \leq j \leq n\right)$ such that $\varepsilon_{v}=\min _{1 \leq j \leq n}\left|\alpha_{v j}\right|>0$, there exists $\Gamma_{v} \subset \Lambda, \Gamma_{v}$ finite such that $\left|\alpha_{\mu j}\right|<\varepsilon_{v}$ for $1 \leq j \leq n$ and for all $\mu \notin \Gamma_{v}$. Hence $\left|\alpha_{\mu j}\right|<\left|\alpha_{v j}\right|$ and $\left|\alpha_{v j}-\alpha_{\mu j}\right|=\left|\alpha_{v j}\right| \geq \varepsilon_{v}$, for $1 \leq j \leq n$ and $\mu \notin \Gamma_{v}$. Therefore, if $\varepsilon<\varepsilon_{v}$, then $W\left(\chi_{v} ; \varepsilon, u_{1}, \ldots, u_{n}\right)=\left\{\chi_{v}\right\}$, that is, $\left\{\chi_{v}\right\}$ is open in $\mathscr{L}(\mathscr{A})$. Hence $\left\{\chi_{v}: v \in \Lambda\right\}$ is a discrete subset of $\mathscr{L}(\mathscr{A})$. On the other hand, if $\chi=\chi_{0}$, then $\chi_{0}\left(u_{j}\right)-\chi_{\mu}\left(u_{j}\right)=-\alpha_{\mu j}$. Hence for $\varepsilon>0$, there exists a finite subset $\Gamma_{\varepsilon}$ of $\Lambda$ such that for $\mu \notin \Gamma_{\varepsilon},\left|\chi_{0}\left(u_{j}\right)-\chi_{\mu}\left(u_{j}\right)\right|=\left|\alpha_{\mu j}\right|<\varepsilon$ for each $1 \leq j \leq n$. In other words, $W\left(\chi_{0} ; \varepsilon, u_{1}, \ldots, u_{n}\right)=\left\{\chi_{\mu}: \mu \notin \Gamma_{\varepsilon}\right\}$. Furthermore, $\chi_{0}=\lim _{\mu \in \Lambda} \chi_{\mu}$ in $\mathscr{L}(\mathscr{A})$ for the weak*-topology. It follows that $\mathscr{X}(\mathscr{A})$ is weak*-compact. Consider on $\Lambda_{0}=\Lambda \cup\{0\}$ the topology such that $\Lambda$ is a discrete subset of $\Lambda_{0}$ and the neighborhoods of 0 are $W_{\Gamma}(0)=\Lambda_{0} \backslash \Gamma$, where $\Gamma \subset \Lambda$ is finite. It becomes clear that $\Lambda_{0}$ is homeomorphic to the Alexandroff compactification of the discrete space $\Lambda$. Identifying $\mathscr{L}(\mathscr{A})$ with $\Lambda_{0}$, we conclude the proof of the proposition.

4.2. Let $\mathscr{C}(\mathscr{C}(\mathscr{A}), \mathbb{K})$ be the $\mathbb{K}$-Banach algebra of the continuous functions $f$ on the compact space $\mathscr{L}(\mathscr{A})$ with values in $\mathbb{K}$. It is readily seen that $f \in \mathscr{C}(\mathscr{L}(\mathscr{A}), \mathbb{K})$ is defined by the family $\left(f\left(\chi_{v}\right): v \in \Lambda_{0}\right) \subset \mathbb{K}$ such that $\lim _{v \in \Lambda} f\left(\chi_{v}\right)=f\left(\chi_{0}\right)$. Hence $\mathscr{C}(\mathscr{C}(\mathscr{A}), \mathbb{K})$ is isometrically isomorphic to the algebra $c_{v}\left(\Lambda_{0}, \mathbb{K}\right)=\left\{a: a=\left(a_{v}: v \in\right.\right.$ $\left.\left.\Lambda_{0}\right) \subset \mathbb{K} ; \lim _{v \in \Lambda} a_{v}=a_{0}\right\}:$ on $c_{v}\left(\Lambda_{0}, \mathbb{K}\right)$, we consider the usual multiplication defined pointwise and the norm $\left\|\left(a_{v}: v \in \Lambda_{0}\right)\right\|=\sup _{v \in \Lambda_{0}}\left|a_{v}\right|$.

COROLLARY 4.11. The Banach algebra $A$ with the unit element id is isometrically isomorphic to the algebra $c_{v}\left(\Lambda_{0}, \mathbb{K}\right)=\left\{a: a=\left(a_{v}\right)_{v \in \Lambda_{0}} \subset \mathbb{K} ; \lim _{v \in \Lambda} a_{v}=a_{0}\right\}$.

Proof. Let $\mathscr{G}: \mathscr{A} \rightarrow \mathscr{C}(\mathscr{X}(\mathscr{A}), \mathbb{K})$ be the Gelfand transform $\mathscr{G}(u)(\chi)=\chi(u)$. As usual,

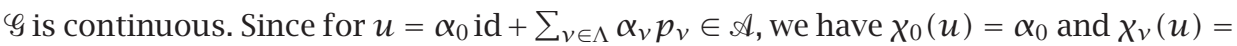
$\alpha_{0}+\alpha_{v}, v \in \Lambda$, and obtain $\|u\|=\max \left(\left|\chi_{0}(u)\right|, \sup _{v \in \Lambda}\left|\chi_{v}(u)\right|\right)=\sup _{\chi \in \mathscr{L}(\mathscr{A})}|\chi(u)|$. Hence, $\|\mathscr{G}(u)\|=\|u\|$. Furthermore, $\mathscr{G}($ id $)(u)=1$, that is, $\mathscr{G}($ id $)=f_{0}$ the constant function equal to 1 . On the other hand, for $v \in \Lambda, \varphi\left(p_{v}\right)(\chi)=1$ if $\chi=\chi_{v}$ and 0 otherwise. Hence, setting for $v \in \Lambda, f_{v}: \mathscr{X}(\mathscr{A}) \rightarrow \mathbb{K}$ such that $f_{v}\left(\chi_{\mu}\right)=\delta_{v, \mu}, \mu \in \Lambda$, we have $\mathscr{G}\left(p_{v}\right)=f_{v}$. Let $u=\alpha_{0}$ id $+\sum_{v \in \Lambda} \alpha_{v} p_{v} \in \mathcal{A}$, we have $\mathscr{G}(u)=\alpha_{0} f_{0}+\sum_{v \in \Lambda} \alpha_{v} f_{v}$. Since any $f \in \mathscr{C}(\mathscr{X}(\mathscr{A}), \mathbb{K})$ can be written in the unique convergent sum $f=f\left(\chi_{0}\right) f_{0}+$ $\sum_{v \in \Lambda}\left(f\left(\chi_{v}\right)-f\left(\chi_{0}\right)\right) f_{v}$ with $\lim _{v \in \Lambda}\left(f\left(\chi_{v}\right)-f\left(\chi_{0}\right)\right)=0$, we have $f=\varphi(u)$ with $u=$

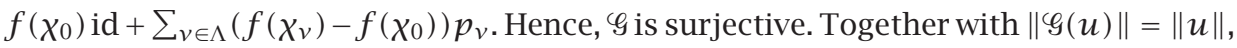
the corollary is proved.

\section{Spectral integration}

5.1. Suppose that $X$ and $Y$ are Banach spaces over a topologically complete nonArchimedean field $\mathbb{K}$ with a nontrivial valuation and $\mathscr{L}(X, Y)$ denotes the Banach space of bounded linear operators $E: X \rightarrow Y$ supplied with the operator norm: $\|E\|:=$ $\sup _{0 \neq x \in X}\|E x\|_{Y} /\|x\|_{X}$. For $X=Y$ we denote $\mathscr{L}(X, Y)$ simply by $\mathscr{L}(X)$. Let $X$ and $Y$ be isomorphic with the Banach spaces $c_{0}(\alpha, \mathbb{K})$ and $c_{0}(\beta, \mathbb{K})$ and let them be supplied 
with the standard orthonormal bases $\left\{e_{j}: j \in \alpha\right\}$ in $X$ and $\left\{q_{j}: j \in \beta\right\}$ in $Y$, respectively, where $c_{0}(\alpha, \mathbb{K}):=\left\{x=\left(x^{j}: j \in \alpha\right) \mid x^{j} \in \mathbb{K}\right.$, such that for each $\epsilon>0$ a set $\{j$ : $\left.\left|x^{j}\right|>\epsilon\right\}$ is finite $\}$ with a norm $\|x\|:=\sup _{j}\left|x^{j}\right|_{\mathbb{K}}, \alpha$ and $\beta$ are ordinals (it is convenient due to Kuratowski-Zorn lemma). Then each operator $E \in \mathscr{L}(X, Y)$ has its matrix realisation $E_{j, k}:=q_{k}^{*} E e_{j}$, which may be infinite, where $q_{k}^{*} \in Y^{*}$ is a continuous $\mathbb{K}$-linear functional $q_{k}^{*}: Y \rightarrow \mathbb{K}$ corresponding to $q_{k}$ under the natural embedding $Y \hookrightarrow Y^{*}$ associated with the chosen basis, $Y^{*}$ is a topologically conjugated or dual space of $\mathbb{K}$-linear functionals on $Y, q_{k}^{*}\left(q_{l}\right)=\delta_{l}^{k}$. Therefore, to each $E \in \mathscr{L}(X, Y)$ there corresponds an adjoint operator $E^{*} \in \mathscr{L}\left(Y^{*}, X^{*}\right)$. By a transposed operator $E^{t}$, we mean a restriction $\left.E^{*}\right|_{Y}$, where $Y$ is embedded into $Y^{*}$ such that $E_{j, k}^{t}=E_{k, j}$ for each $j \in \alpha$ and $k \in \beta$.

This means, that if $X=Y$ and $E^{t}=E$, then $E$ is called a symmetric operator. For $X=Y=c_{0}(\alpha, \mathbb{K})$ there is an inclusion $E^{*}(Y) \subset X^{*}$. Since $X^{*}=l^{\infty}(\alpha, \mathbb{K})$, then $\|x\|_{X}=$ $\|x\|_{X^{*}}$ for each $x \in X$. Since $\|E\|=\sup _{j, k}\left|E_{j, k}\right|$, then $\|E\|=\left\|E^{*}\right\|$ and $\|E\|=\left\|E^{t}\right\|$. If $A, E \in \mathscr{L}(X)$ and $E=A^{t}$, then $A$ and $E$ belong to the closed subalgebra $\mathscr{L}_{0}(X)$ (see Section 2).

5.1.1. Now let $A$ be an abstract Banach algebra over a field $\mathbb{K}$, which is complete relative to a norm $\|*\|_{\mathbb{K}}$ in it. We say that $A$ is with an operation of transposition $a \mapsto a^{t}$ for each $a \in A$ if the following conditions $(\alpha),(\beta),(\gamma)$, and $(\delta)$ are satisfied:

( $\alpha)(a+b)^{t}=a^{t}+b^{t}$;

(B) $(\lambda a)^{t}=\lambda a^{t}$;

( $)(a b)^{t}=b^{t} a^{t}$

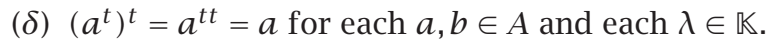

Let $A$ be an algebra over $\mathbb{K}$, which satisfies the following conditions (i), (ii), and (iii):

(i) $A$ is a Banach algebra,

(ii) with the operation of transposition $a \mapsto a^{t}$,

(iii) $\left\|a^{t} a\right\|=\|a\|^{2}$ for each $a \in A$ while evaluation of norms.

Then such algebra is called an $E$-algebra.

Without condition (iii), it is called a $T$-algebra. If instead of (iii) it satisfies the following condition:

(iv) $\left\|a^{t} a\right\|=\left\|a^{2}\right\|$ then $A$ is called an $S$-algebra.

For each $E$-algebra, we have $\|a\|^{2}=\left\|a a^{t}\right\| \leq\|a\|\left\|a^{t}\right\|$, hence $\|a\| \leq\left\|a^{t}\right\|$ and also $\left\|a^{t}\right\| \leq\left\|\left(a^{t}\right)^{t}\right\|=\|a\|$, consequently $\|a\|=\left\|a^{t}\right\|$.

5.1.2. Evidently, $\mathscr{L}_{0}(X)$ is a $T$-algebra. Each $C$-algebra is at the same time an $E$ algebra (see also Section 5.5), since for each singleton $x \in X$ a closed subalgebra $C(\{x\}, \mathbb{K})$ is isomorphic with $\mathbb{K}$ and the restriction of transposition on $C(\{x\}, \mathbb{K})$ gives $f^{t}(x)=f(x)$ for each $f \in C_{\infty}(X, \mathbb{K})$.

Let $A_{1}, \ldots, A_{n}$ be linear operators. Then the equation

$$
\left[\sum_{j=1}^{n} \lambda_{j} A_{j}, \sum_{k=1}^{n} \mu_{k} A_{k}\right]=\sum_{j<k}\left(\lambda_{j} \mu_{k}-\lambda_{k} \mu_{j}\right)\left(A_{j} A_{k}-A_{k} A_{j}\right)=0,
$$

for each $\lambda_{j}$ and $\mu_{k} \in \mathbb{K}$, is equivalent to $\left[A_{j}, A_{k}\right]=0$ for each $j<k$. In view of [15, 16, Sections IV.6, 7, VII.7, and VIII.2] for each $n \in \mathbb{N}$ there are pairwise commuting 
matrices of the size $m \times m$ such that for sufficiently large $m>n$ they in addition can be found nondiagonal (nonreducible to diagonal form by transformations, $U_{j} A_{j} U_{j}^{-1}$, where $U_{j}$ are invertible matrices), since in view of $[15,16$, Theorem VIII.7.2] a number of linearly independent matrices, which commute with the given matrix $A$ is defined by the following formula: $N=n_{1}+3 n_{2}+\cdots+(2 t-1) n_{t}$, where $n_{1}, n_{2}, \ldots, n_{t}$ are degrees of nonconstant invariant polynomials $i_{1}(\lambda), i_{2}(\lambda), \ldots, i_{t}(\lambda)$ and $n=n_{1}+n_{2}+\cdots+n_{t}$ is a size of the square $n \times n$ matrix $A$. This can be done by suitable choices of Jordan forms of matrices over $\mathbb{K}$. From this it follows that in $\mathscr{L}_{0}\left(c_{0}\left(\omega_{0}, \mathbb{K}\right)\right)$ for each $n \in \mathbb{N}$, there always exist $n$ pairwise commuting operators such that they are not reducible to the diagonal form by adjoint transformations $U_{j} A_{j} U_{j}^{-1}$. This produces examples of $T$-algebras. When in the (finite case) Jordan form $\left|\lambda_{j, k}\right|>1$ and $\left\|I-U_{j}\right\|<1$ for each $j=1, \ldots, n$, where $\lambda_{j, k}$ are diagonal elements of the Jordan normal forms of $A_{j}$, then each $A_{j}$ together with $A_{j}^{t}$ satisfy condition (iii). We take the case $U_{j}=U_{1}=: U$ for each $j$. Let in addition $A_{j}$ be pairwise commutative and have block forms $(v)$ $A_{j}=\operatorname{diag}\left(A_{j, 1}, \ldots, A_{j, n}\right), A_{j, l}$ are $m_{l} \times m_{l}$ square matrices and $A_{j, l}=0$ for each $l \neq j$ and $A_{j, j} \neq 0$. Consider their transposed matrices also, then the linear span of all their products $B_{j_{1}} \cdots B_{j_{s}}$ produces commutative $E$-algebra, which is generated by nondiagonalizable over $\mathbb{C}_{p}$ matrices, where $B_{j}$ is equal either to $A_{j}$ or to $A_{j}^{t}$, since $\left|\lambda_{j, k}\right|^{a}>$ $\left|b \lambda_{j, k}\right|^{c}$ for each $a>c>0$ and $b \in \mathbb{Z}$. As it follows from [15, 16, Section VIII.7.2] these $A_{j}$ can be chosen such that $\operatorname{sp}_{\mathbb{K}}\left\{I, A_{j}, A_{j}^{2}, \ldots, A_{j}^{m}\right\}$ does not contain $A_{l}$ for each $l \neq j$.

The construction of $S$-algebras can be done analogously and more lightly, since condition (iii) is replaced by condition (iv).

Let $A$ be an $E$-algebra with a $\mathbb{K}$-linear isometry $Y: A \rightarrow A$ such that $Y(a b)=$ $Y(b) Y(a)$ and $Y^{t}(a)=Y\left(a^{t}\right)$ for each $a, b \in A$. Then $Y(A)$ is an $E$-algebra.

There are general constructions of Banach algebras also. In particular, we can take a free Banach algebra $A$ generated by a set $J$. This means, that $A$ is a completion of $\operatorname{sp}_{\mathbb{K}}\left\{a_{1} \cdots a_{n}: a_{1}, \ldots, a_{n} \in J, n \in \mathbb{N}\right\}$ with the definite order of letters $a_{1}, \ldots, a_{n}$ in each word $w=a_{1} a_{2} \cdots a_{n}$, when neighbouring elements $a_{j}$ and $a_{j+1}$ are distinct in $J$. There exists a norm on $\operatorname{sp}_{\mathbb{K}}\left\{a_{1} \cdots a_{n}: a_{1}, \ldots, a_{n} \in J, n \in \mathbb{N}\right\}$ such that $\|a b\| \leq\|a\|\|b\|$ for each $a, b \in A$. For example, $\|w\|=1$ for each word $w=a_{1} \cdots a_{n}$ with $a_{1}, \ldots, a_{n} \in$ $J,\left\|c_{1} w_{1}+\cdots+c_{m} w_{m}\right\|=\max _{1 \leq j \leq m}\left|c_{j}\right|_{\mathbb{K}}$ for different words $w_{1}, \ldots, w_{m}$ with $c_{j} \in \mathbb{K}$ for each $j=1, \ldots, m$. Then for $Y: A \rightarrow A$ preserving a closed ideal $V$ we can consider the quotient mapping $\bar{Y}: \bar{A} \rightarrow \bar{A}$, where $\bar{A}=A / V$ and $Y$ on $A$ is defined by $Y$ on $J$ due to the continuous extension.

Another example is the following. For a subset $J$ of symmetric (i.e., $a^{t}=a$ for each $\left.a \in J \subset \mathscr{L}_{0}(X)\right)$ pairwise commuting elements (i.e., $a b=b a$ for each $a$ and $b \in J \subset$ $\left.\mathscr{L}_{0}(X)\right)$ let $A:=\operatorname{cl}\left(\operatorname{sp}_{\mathbb{K}}\left\{\prod_{i=1}^{m} a_{i}^{n_{i}}: 0 \leq n_{i} \in \mathbb{Z}, m \in \mathbb{N}, a_{i} \in J\right\}\right)$, where $a^{0}:=I$ is the unit operator on $X$. Then such $A$ is a $T$-algebra. Since $\left\|a^{t} a\right\|=\left\|a^{2}\right\|$ for each $a \in A$, then it is an $S$-algebra.

Another example of an $E$-algebra is the algebra of diagonal operators in $\mathscr{L}_{0}(X)$. Then each $E$-algebra is certainly an $S$-algebra and each $S$-algebra is a $T$-algebra (see also Lemmas 4.5, 4.8 and Proposition 4.10(i)). Above were constructed more interesting examples of $E$-algebras and $S$-algebras. In general, diagonal form of an algebra is unnecessary for the spectral theory. Moreover, there are well-known theorems, when Lie algebras (in particular of finite square $m \times m$ matrices over $\mathbb{C}_{p}$ ) can be reduced 
simultaneously to the upper triangular form by one transformation $U A_{j} U^{-1}$ (see [17, Iwasawa Theorem 4.7.2]). There are also cases, when it may be over $\mathbb{K}$. Using limits such cases can be spread on subalgebras of $\mathscr{L}_{0}(X)$.

It will be shown below that for the spectral integration it is sufficient to consider C-algebras.

5.2. Let $A$ and $B$ be two $E$-algebras over the same field $\mathbb{K}$, an algebraic homomorphism $T: A \rightarrow B$ is called a $t$-representation of $A$ in $B$, if $T_{a^{t}}=\left(T_{a}\right)^{t}$ for each $a \in A$. The reducing ideal $Y$ of $A$ is defined as the intersection of the kernels of all $t$-representations of $A$. The reducing ideal $\Upsilon$ is also called the $t$-radical. If $\Upsilon=0$, then $A$ is called reduced (or $t$-simple).

Let $\|a\|_{t}:=\sup _{T \in \Psi}\|T a\|$ for a reduced algebra $A$, where $\Psi:=\Psi_{A}$ denotes the family of all $t$-representations of $A$. Since $A$ is reduced, then $\|a\|_{t} \neq 0$ for each $A \ni a \neq 0$. Such $\|*\|_{t}$ is called an $E$-norm of $A$.

The $E$-algebra obtained by completing $A / Y$ by its $E$-norm is called the $E$-completion of $A$ and is denoted by $A_{t}$. Denote by $\pi: A \rightarrow A / Y$ the natural $t$-homomorphism of $A$ into $A_{t}$ such that $\pi(a)=a+Y$ for each $a \in A$. Then the map $T \mapsto T^{\prime}=T \circ \pi$ is a bijective correspondence between the set of all $t$-representations $T$ of $A_{t}$ and the set of all $t$-representations $T^{\prime}$ of $A$. This correspondence preserves closed stable subspaces, nondegeneracy, bounded intertwining operators, isometric equivalence and Banach direct sums.

5.3. Let $A$ be a commutative Banach $T$-algebra and $A^{+}$denotes the Gelfand space of $A$, that is, $A^{+}=\operatorname{Sp}(A)$, where $\operatorname{Sp}(A)$ was defined in [28, Chapter 6], it is the set of all nonzero algebra homomorphisms $\phi: A \rightarrow \mathbb{K}$ topologized as the subset of $\mathbb{K}^{A}$. Every $x \in A$ induces a function $G_{x}: \operatorname{Sp}(A) \rightarrow \mathbb{K}$ by $G_{x}(\phi):=\phi(x)$, where $\phi \in \operatorname{Sp}(A), G_{x}$ is called the Gelfand transform of $x, G$ is called the Gelfand transformation. Then it is defined the spectral norm $\|x\|_{\mathrm{sp}}:=\sup _{\phi \in \operatorname{Sp}(A)}\left|G_{x}(\phi)\right|$ of $x \in A$. If $\operatorname{Sp}(A)=\varnothing$, then $\|x\|_{\mathrm{sp}}:=0$ for each $x \in A$. We denote by $\hat{A}$ the closed subset of $A^{+}$consisting of those $\phi \in A^{+}$for which $\phi\left(a^{t}\right)=\phi(a)$ for each $a \in A, \phi \in A$ is called symmetric, if $\phi \in \hat{A}$. Let $C_{\infty}(\hat{A}, \mathbb{K})$ be the same space as in [28]. For a locally compact $E$ the space $C_{\infty}(E, \mathbb{K})$ is a subspace of the space $\operatorname{BUC}(E, \mathbb{K})$ of bounded uniformly continuous functions $f: E \rightarrow \mathbb{K}$ such that for each $\epsilon>0$ there exists a compact subset $V \subset E$ for which $|f(x)|<\epsilon$ for each $x \in E \backslash V$. When $E$ is not locally compact and has an embedding into $B(\mathbb{K}, 0,1)^{\gamma}$ such that $E \cup\left\{x_{0}\right\}=\operatorname{cl}(E)$ we put $C_{\infty}(E, \mathbb{K}):=\left\{f \in C(E, \mathbb{K}): \lim _{x \rightarrow x_{0}} f(x)=0\right\}$, where $B(X, x, r):=\{y \in X: d(x, y) \leq r\}$ is a ball in the metric space $(X, d), \operatorname{cl}(E)$ is taken in $B(\mathbb{K}, 0,1)^{\gamma}, \gamma$ is an ordinal, $x_{0} \in B(\mathbb{K}, 0,1)^{\gamma}$.

Definition 5.1 (see also [28, Chapter 6]). A commutative Banach algebra $A$ is called a $C$-algebra if it is isomorphic with $C_{\infty}(X, \mathbb{K})$ for a locally compact Hausdorff hereditarily disconnected space $X$, where $f+g$ and $f g$ are defined pointwise for each $f, g \in C_{\infty}(X, \mathbb{K})$.

Proposition 5.2. The reducing ideal $Y$ of $A$ consists of those $a \in A$ such that $\hat{a}(\phi)=$ 0 for each $\phi \in \hat{A}$. The equation

(i) $F(\pi(a))=\left.\hat{a}\right|_{\hat{A}}$ determines an isometric $t$-isomorphism $F$ of $A_{t}$ onto $C_{\infty}(\hat{A}, \mathbb{K})$, when $\mathbb{K}$ is a locally compact field. 
Proof. We have $\sup _{\phi \in \hat{A}}|\hat{a}(\phi)| \leq\|\pi(a)\|_{t}$ for each $a \in A$. Then we take a $t$ representation $T$ of $A$ and $B:=\operatorname{cl}_{\|} \| \operatorname{range}(T)$, hence $B$ is a commutative $E$-algebra. To finish the proof of Proposition 5.2 we need the following.

Proposition 5.3. Let $A$ be a commutative E-algebra as in Section 5.1: $A=$ $\operatorname{cl}\left(\operatorname{sp}_{\mathbb{K}}\left\{\prod_{i=1}^{m} a_{i}^{n_{i}}: 0 \leq n_{i} \in \mathbb{Z}, a_{i} \in J, a^{t}=a, m \in \mathbb{N}\right\}\right)$, then $\hat{A}=A^{+}$. Furthermore, the Gelfand transform map $a \mapsto \hat{a}$ is an isometric isomorphism of $A$ onto $C_{\infty}(\hat{A}, \mathbb{K})$, when $\mathbb{K}$ is a locally compact field.

Proof. In view of [28, Corollaries 6.13, 6.14, and 6.17] it is sufficient to show that $A^{+}=\hat{A}$, since $A$ is isomorphic with $C_{\infty}(\operatorname{Sp}(A), \mathbb{K})$, where $\operatorname{Sp}(A)=\hat{A}$. If $a^{t}=a \in A$ and $\phi \in A^{+}$, then $\phi\left(a^{t}\right)=\phi(a) \in \mathbb{K}$. If $\mathbf{1} \notin A$, then $\phi$ extends to a $t$-homomorphism of the $S$-algebra $A_{1}$ obtained by adjoining the unit 1 to $A$, since it is possible to consider $X \oplus \mathbb{K}$ (cf., about adjoining of 1 in [14, 13, Section VI.3.10] and [28, Chapter 6]). Since $\mathbb{K}$ is locally compact, $A_{1}$ is isomorphic with $C(\alpha Y, \mathbb{K})$, where $\alpha Y=\operatorname{Sp}(A) \cup\{0\}$ is a one-point (Alexandroff) compactification of $\operatorname{sp}(A)$ (see [28, Observation 6.2]). Indeed, $\|a\|=\sup _{\chi \in A^{+}}|\chi(a)|$. Let $\phi(a)=r \in \mathbb{K}, b:=a+z \mathbf{1}$, where $z \in \mathbb{K}$. From $\|\phi\| \leq 1$ it follows that $\left|\phi\left(b^{t} b\right)\right|_{p}=\left|(r+z)^{2}\right|_{p} \leq\left\|b^{2}\right\|$ and $\left\|b^{2}\right\|=\left\|a^{t} a+z a^{t}+z a+z^{2} \mathbf{1}\right\| \leq$ $\max \left(\left\|a^{2}\right\|,|z|_{p}\|a\|,|z|_{p}^{2}\right)$. Then there exists $0<\epsilon<\left(\left\|a^{2}\right\|\right)^{1 / 2}$ such that for each $|z|_{p}<$ $\epsilon:\left|\phi\left(b^{t} b\right)\right|_{p} \leq\left\|a^{2}\right\|$, consequently $\phi$ has the continuous extension on $A_{1}$.

If $\phi \in A^{+}$and $a=b+c \in A$ with $b^{t}=b$ and $c^{t}=-c \in A$, then $\phi\left(a^{t}\right)=\phi(b)-\phi(c)$. If $\phi\left(a^{t}\right)=-\phi(a)$ for each $a \in A$, then $\phi(b)=0$ for each $b=b^{t} \in A$. If $\phi\left(a^{t}\right)=$ $\phi(a)$ for each $a \in A$, then $\phi(c)=0$ for each $c^{t}=-c \in A$. The operation of transposition $a \mapsto a^{t}$ is continuous in $A$. Let $\phi \in A^{+}$and $\phi \neq 0$. Therefore, for each $\phi(a) \neq 0$ we have $\phi\left(a^{t}\right) \neq 0$, since $a^{t t}=a$. Hence $\phi\left(a^{t}\right)=\lambda_{\phi} \phi(a)$ for each $a \in A$ such that $\phi(a) \neq 0$, since coker $\mathbb{K}_{\mathbb{K}} \phi$ is one-dimensional, where $0 \neq \lambda_{\phi} \in \mathbb{K}$. We have $\phi\left(\left(a^{t} a\right)^{n}\right)=\lambda_{\phi}^{n} \phi(a)^{2 n}$. Since $\left\|a^{t} a\right\|=\left\|a^{2}\right\|, a^{t t}=a$, and $\phi$ is the continuous multiplicative linear functional, then $\left|\lambda_{\phi}\right|_{p}=1$. On the other hand, $\lambda_{\phi} \phi(a b)=\phi\left(a^{t} b^{t}\right)=$ $\phi\left(a^{t}\right) \phi\left(b^{t}\right)=\lambda_{\phi}^{2} \phi(a) \phi(b)=\lambda_{\phi} \phi(a b)$, hence $\lambda_{\phi}=1$, where there are $a$ and $b \in A$ such that $\phi(a b) \neq 0$. Therefore, $\phi^{t}=\phi$, where $\phi^{t}(a):=\phi\left(a^{t}\right)$ for each $a \in A$. Consequently, $\hat{A}=A^{+}$.

Continuation of THe Proof of Proposition 5.2. In view of Section 2 there exists $\psi \in \hat{B}$ such that $\left|\psi\left(T_{a}\right)\right|=\left\|T_{a}\right\|$, since $\psi^{\prime}: a \mapsto \psi\left(T_{a}\right) \in \hat{A}$, hence $\left\|T_{a}\right\|=\left|\psi^{\prime}(a)\right| \leq$ $\sup _{\phi \in \hat{A}}|\hat{a}(\phi)|$, consequently, $\|\pi(a)\|_{t} \leq \sup _{\phi \in \hat{A}}|\hat{a}(\phi)|$. Therefore, $\|\pi(a)\|_{t}=$ $\sup _{\phi \in \hat{A}}|\hat{a}(\phi)|$, hence the map $F$ defines the isometric $t$-isomorphism of $A_{t}$ into $C_{\infty}(\operatorname{Sp}(A), \mathbb{K})$. The range of $F$ is a $T$-subalgebra of $C_{\infty}(\hat{A}, \mathbb{K})$, which automatically separates points of $\hat{A}$, consequently, by the Kaplansky theorem cl $\operatorname{range}(F)=C_{\infty}(\hat{A}, \mathbb{K})$ (see [25, Section A.4]).

5.4. Let $H=c_{0}(\alpha, \mathbb{K})$, where $\mathbb{K}$ is a topologically complete field. A strong operator topology in $\mathscr{L}(H, Y)$ (see Section 1 ) is given by a base $V_{\epsilon ; E ; x_{1}, \ldots, x_{n}}:=\{Z \in \mathscr{L}(H, Y)$ : $\left.\sup _{1 \leq j \leq n}\left\|(E-Z) x_{j}\right\|_{Y}<\epsilon\right\}$, where $0<\epsilon, E \in \mathscr{L}(H, Y), x_{j} \in H ; j=1, \ldots, n ; n \in \mathbb{N}$. Let $X$ be a topological space with the small inductive dimension $\operatorname{ind}(X)=0$. An $H$-projection-valued measure on an algebra $\mathbf{L}$ of subsets of $X$ is a function $P$ on L assigning to each $A \in \mathbf{L}$ a projection $P(A)$ on $H$ and satisfying the following conditions: 
(i) $P(X)=\mathbf{1}_{H}$,

(ii) for each sequence $\left\{A_{n}: n=1, \ldots, k\right\}$ of pairwise disjoint sets in $\mathbf{L}$ there are projections $P\left(A_{n}\right)$ such that $P\left(A_{n}\right) P\left(A_{l}\right)=0$ for each $n \neq l$ and $P\left(\bigcup_{n=1}^{k} A_{n}\right)=$ $\sum_{n=1}^{k} P\left(A_{n}\right)$,

(iii) if $\mathbf{A} \subset \mathbf{L}$ is shrinking and $\cap \mathbf{A}=\varnothing$, then $\lim _{A \in \mathbf{A}} P(A)=0$, where the convergence on the righthand side is unconditional in the strong operator topology and the sum is equal to the projection onto the closed linear span over $\mathbb{K}$ of $\left\{\operatorname{range}\left(P\left(A_{n}\right)\right): n=1, \ldots, k\right\}$ such that $P(\varnothing)=0, k \in \mathbb{N}$.

If $\eta \in H^{*}$ and $\xi \in H$, then $A \mapsto \eta(P(A) \xi)$ is a $\mathbb{K}$-valued measure on $\mathbf{L}$. The case of a $\sigma$-algebra $\mathbf{L}$ and of $k=\infty$ in (ii) is unnecessary for the subsequent consideration and it will not be used, but it may be considered as a particular case. The $\sigma$-additive case leads to the restriction that each measure $\eta(P(A) \xi)$ is atomic, when $\mathbb{K}$ is spherically complete (see [28, Chapter 7]).

Then by definition $P(A) \leq P(B)$ if and only if the range $(P(A)) \subset$ the range $P(B)$. There are many projection operators on $H$, but for $P$ there is chosen some such fixed system.

A subset $A \subset X$ is called $P$-null if there exists $B \in \mathbf{L}$ such that $A \subset B$ and $P(B)=0$, $A$ is called $P$-measurable if $A \triangle B$ is $P$-null, where $A \triangle B:=(A \backslash B) \cup(B \backslash A)$. A function $f: X \rightarrow \mathbb{K}$ is called $P$-measurable, if $f^{-1}(D)$ is $P$-measurable for each $D$ in a field $B \operatorname{co}(\mathbb{K})$ of clopen subsets of $\mathbb{K}$. It is essentially bounded, if there exists $k>0$ such that $\{x:|f(x)|>k\}$ is $P$-null, $\|f\|_{\infty}$ is by definition the infimum of such $k$. Then $\mathscr{F}:=\mathrm{sp}_{\mathbb{K}}\left\{\mathrm{Ch}_{B}: B \in \mathbf{L}\right\}$ is called the space of simple functions, where $\mathrm{Ch}_{B}$ denotes the characteristic function of $B$. The completion of $\mathscr{F}$ relative to $\|*\|_{\infty}$ is the Banach algebra $L_{\infty}(P)$ under the pointwise multiplication.

There exists a unique linear mapping $\mathscr{I}: \mathscr{F} \rightarrow \mathscr{L}(H)$ by the following formula:

(iv) $\mathscr{I}\left(\sum_{i=1}^{n} \lambda_{i} \mathrm{Ch}_{B_{i}}\right)=\sum_{i=1}^{n} \lambda_{i} P\left(B_{i}\right)$, where $n \in \mathbb{N}, B_{i} \in \mathbf{L}, \lambda_{i} \in \mathbb{K}$. Since

(v) $\|\mathscr{I}(f)\|=\|f\|_{\infty}$, then $\mathscr{I}$ extends to a linear isometry (also called $\mathscr{I}$ ) of $L_{\infty}(P)$ onto $\mathscr{L}(H)$.

If $f \in L_{\infty}(P)$, then the operator $\mathscr{I}(f)$ in $\mathscr{L}(H)$ is called the spectral integral of $f$ with respect to $P$ and is denoted

(vi) $\int_{X} f(x) P(d x):=\mathscr{I}(f)$.

Evidently properties (I), (II), (III), (V), and (VI) from [14, 13, Section II.11.8] are transferable onto the case considered here. These and other properties of the spectral integral are as follows.

Proposition 5.4. A spectral integral has the following properties:

(I) $\int_{X} f(x) P(d x)=\int_{X} g(x) P(d x)$ if and only if $f$ and $g$ differ only on a $P$-null set;

(II) $\int_{X} f(x) P(d x)$ is linear in $f$;

(III) $\int_{X} f(x) g(x) P(d x)=\left(\int_{X} f(x) P(d x)\right)\left(\int_{X} g(x) P(d x)\right)$, for each $f$ and $g \in$ $L_{\infty}(P)$;

(IV) $\left\|\int_{X} f(x) P(d x)\right\|=\|f\|_{\infty}$;

(V) if $A \in \mathbf{L}$, then $\int_{X} C h_{A}(x) P(d x)=P(A)$, in particular $\int_{X} P(d x)=P(X)=\mathbf{1}_{H}$;

(VI) for each pair $\xi \in H$ and $\eta^{*} \in H^{*}$, let $\mu_{\xi, \eta}(A):=\eta^{*}(P(A) \xi)$ for each $A \in \mathbf{L}$. If $E=\int_{X} f(x) P(d x)$ then $\eta^{*}(E \xi)=\int_{X} f(x) \mu_{\xi, \eta}(d x) ;$ 
(VII) if $A \in \mathbf{L}$, then $P(A)$ commutes with $\int_{X} f(x) P(d x)$, where $e^{i}:=e_{i}^{*}$ such that $e^{i}\left(e_{j}\right)=\delta_{j}^{i}$.

An $H$-projection-valued measure $P$ on the algebra $\mathbf{L}$ containing an algebra $B c o(X)$ of clopen (closed and open at the same time) subsets of $X$ is called an $H$-projectionvalued tight measure on $X$. We call $P$ regular if

(vii) $P(A)=\sup \{P(C): C \subset A$ and $C$ is compact $\}$ for each $A \in \mathbf{L}$, where sup is the least closed subspace of $H$ containing range $P(C)$ and to it corresponds a projector on this subspace. Indeed, $P(A) H$ is closed in $H$, since $P^{2}(A)=P(A)$. Therefore,

(viii) $P(A)=\inf \{P(U): U$ is open and $U \supset A\}=I-\sup \{P(C): C \subset X \backslash A$ and $C$ is compact $\}$, hence

(ix) the infimum corresponds to the projection on $\bigcap_{U \supset A, U \text { is open }} P(U) H$.

A measure $\mu: \mathbf{L} \rightarrow \mathbb{K}$ is called regular, if for each $\epsilon>0$ and each $A \in \mathbf{L}$ with $\|A\|_{\mu}<\infty$ there exists a compact subset $C \subset A$ such that $\|A \backslash C\|_{\mu}<\epsilon$. Since $\|P(X)\|=1$, then $\left\|\mu_{\xi, \eta}\right\| \leq\|\xi\|_{H}\|\eta\|_{H^{*}}$. For the space $H$ over $\mathbb{K}$, measures $\mu_{\xi, \eta}$ on locally compact $X$ are tight for each $\xi, \eta$ in a subset $J \subset H \hookrightarrow H^{*}$ separating points of $H$ if and only if $P$ is defined on $\mathbf{L} \supset B \operatorname{co}(X) ; P$ is regular if and only if $\mu_{\xi, \eta}$ are regular for each $\xi, \eta \in J$ due to Conditions (viii) and (ix). We can restrict our consideration by $\mu_{\xi, \xi}$ instead of $\mu_{\xi, \eta}$ with $\xi, \eta \in \mathrm{sp}_{\mathbb{K}} J$, since $\left({ }_{-}^{+}\right) 2 \mu_{\xi, \eta}=\mu_{\xi\left({ }_{-}^{+}\right) \eta, \xi\left({ }_{-}^{+}\right) \eta}-\mu_{\xi, \xi}-\mu_{\eta, \eta}$.

By the closed support of an $H$-projection-valued tight measure $P$ on $X$ we mean the closed set $D$ of all those $x \in X$ such that $P(U) \neq 0$ for each open neighbourhood $x \in U, \operatorname{supp}(P):=D$.

5.5. We fix a locally compact totally disconnected Hausdorff space $X$ and a Banach space $H$ over $\mathbb{K}$ and let $T: C_{\infty}(X, \mathbb{K}) \rightarrow \mathscr{L}(H)$ be a linear continuous map from the $C$-algebra $C_{\infty}(X, \mathbb{K})$ of functions $f: X \rightarrow \mathbb{K}$ such that:

(i) $T_{f g}=T_{f} T_{g}$ for each $f$ and $g \in C_{\infty}(X, \mathbb{K})$,

(ii) $T_{1}=I$ for compact $X$.

In general, $C_{\infty}(X, \mathbb{K})$ can be considered as an $E$-algebra if we define $f^{t}:=f$ for each $f \in C_{\infty}(X, \mathbb{K})$, so we can put $T_{f}^{t}=T_{f}$, but the latter equality will not be used.

From this definition it follows, that $\|T\| \leq 1$, since $T_{f n}=T_{f}^{n}$ for each $n \in \mathbb{Z}$ and $f \in C_{\infty}(X, \mathbb{K})$. If $X$ is locally compact and is not compact, then $X_{\infty}:=X \cup\left\{x_{\infty}\right\}$ be its one-point Alexandroff compactification. Each $f \in C\left(X_{\infty}, \mathbb{K}\right)$ can be written just in one way in the form $f=\lambda \mathbf{1}+g$, where $g \in C_{\infty}(X, \mathbb{K})$ and $\mathbf{1}$ is the unit function on $X_{\infty}$. Therefore, we can extend $T: C_{\infty}(X, \mathbb{K}) \rightarrow \mathscr{L}(H)$ to a linear map $T^{\prime}: C\left(X_{\infty}, \mathbb{K}\right) \rightarrow \mathscr{L}(H)$ by setting $T_{\lambda \mathbf{1}+g}^{\prime}=\lambda \mathbf{1}_{H}+T_{g}$ such that $T_{\mathbf{1}}^{\prime}=\mathbf{1}_{H}$.

Therefore, $f \mapsto \eta^{*}\left(T_{f} \xi\right)=: \tilde{\mu}_{\xi, \eta}(f)$ is a continuous $\mathbb{K}$-linear functional on $C_{\infty}(X, \mathbb{K})$, where $\xi \in H$ and $\eta^{*} \in H^{*}$. In view of [28, Theorems 7.18 and 7.22] about correspondence between measures and continuous linear functionals (the non-Archimedean analog of the Riesz representation theorem) there exists the unique measure $\mu_{\xi, \eta} \in$ $M(X)$ such that

(I) $\eta^{*}\left(T_{f} \xi\right)=\int_{X} f(x) \mu_{\xi, \eta}(d x)$ for each $f \in C_{\infty}(X, \mathbb{K})$. In case $T_{f}^{t}=T_{f}$ we have $\mu_{\xi, \eta}=\mu_{\eta, \xi}$, when $\xi, \eta \in H$. Since $T_{1}=I$, then $\mu_{\xi, \eta}(X)=\eta^{*}(\xi)=\xi^{*}(\eta)$. Then for each $A \in \mathbf{L},\|A\|_{\mu_{\xi, \eta}} \leq\|\xi\|\|\eta\| \sup _{f \neq 0}\left\|T_{f}\right\| \leq\|\xi\|\|\eta\|$. Since $H$ considered 
as a subspace of $H^{*}$ separates points in $H$, then for each $A \in \mathbf{L}$ there exists a unique linear operator $P(A) \in \mathscr{L}(H)$ such that

(II) $\|P(A)\| \leq 1$ and $\eta^{*}(P(A) \xi)=\mu_{\xi, \eta}(A)$, since $\mu_{\xi, \eta}(A)$ is a continuous bilinear $\mathbb{K}$-valued functional by $\xi$ and $\eta \in H$. From the existence of the $H$-projectionvalued measure in the case of locally compact $X$, we get a projection-valued measure $P^{\prime}$ on $X_{\infty}$ such that

(III) $T^{\prime}{ }_{f}=\int_{X_{\infty}} f(x) P^{\prime}(d x)$ for each $f \in C\left(X_{\infty}, \mathbb{K}\right)$.

LEMMA 5.5. For each $A$ and $B \in \mathbf{L}$, (i) $P(A \cap B)=P(A) P(B)=P(B) P(A)$.

Proof. For each $g \in C_{\infty}(X, \mathbb{K})$ and $\xi, \eta \in H$ let $\nu_{g}(d x):=g(x) \mu_{\xi, \eta}(d x)$. For each $f$ and $g \in C_{\infty}(X, \mathbb{K})$ we have: $\int_{X} f(x) \mu_{T_{g} \xi, \eta}(d x)=\eta^{*}\left(T_{f} T_{g} \xi\right)=\int_{X} f(x) g(x) \mu_{\xi, \eta}(d x)=$ $\int_{X} f(x) \nu_{g}(d x)$, consequently $\nu_{g}=\mu_{T_{g} \xi, \eta}$. For a fixed $A \in \mathbf{L}$ let $\rho(B):=\mu_{\xi, \eta}(A \cap B)$ for each $B \in \mathbf{L}$. Therefore, $\rho$ is a tight measure on $X: \rho \in M(X)$, where $M(X)$ denotes the set of all tight measures on $X$. For each $g \in C_{\infty}(X, \mathbb{K})$ there are equalities

$$
\int_{X} g(x) \rho(d x)=\int_{A} g(x) \mu_{\xi, \eta}(d x)=v_{g}(A)=\int_{X} g(x) \mu_{P(A) \xi, \eta}(d x) .
$$

Then for each $B \in \mathbf{L}$, we get

$$
\eta^{*}(P(A \cap B) \xi)=\mu_{\xi, \eta}(A \cap B)=\rho(B)=\mu_{P(A) \xi, \eta}(B)=\eta^{*}(P(A) P(B) \xi) .
$$

The elements $\xi$ and $\eta \in H$ were arbitrary, hence $P(A \cap B)=P(A) P(B)$. Interchanging $A$ and $B$ we get the conclusion of this lemma.

COROLlary 5.6. For each $A \in \mathbf{L}$ we have $P^{2}(A)=P(A)$ and $P(A)$ is a projection operator such that $P(X)=I$. If $A \cap B=\varnothing, A$ and $B \in \mathbf{L}$, then $P(A) P(B)=0$.

Proposition 5.7. If conditions (i), (ii), (iii) of Section 5.4 are satisfied, then $P$ is the unique regular $H$-projection-valued tight measure on $X$ and $T_{f}=\int_{X} f(x) P(d x)$ for each $f \in C_{\infty}(X, \mathbb{K})$.

Note 5.8. Such integral is called the spectral integral.

Proof. Let $\left\{A_{n}: n \in \mathbb{N}\right\}$ be a sequence of pairwise disjoint subsets of $X, A_{n} \in \mathbf{L}$. Since $X$ is locally compact, then the spectral integral defined in Section 5 as the limit of certain finite sums exists. By Corollary 5.6, $P\left(A_{n}\right)$ are pairwise orthogonal projectors. Put $Q=\sum_{n} P\left(A_{n}\right)$. Then for each $\xi$ and $\eta \in H$, we have $\eta^{*}(Q \xi)=\sum_{n} \eta^{*}\left(P\left(A_{n}\right) \xi\right)=$ $\sum_{n} \mu_{\xi, \eta}\left(A_{n}\right)=\mu_{\xi, \eta}\left(\bigcup_{n} A_{n}\right)=\eta^{*}\left(P\left(\bigcup_{n} A_{n}\right) \xi\right)$, consequently, $P\left(\bigcup_{n} A_{n}\right)=\sum_{n} P\left(A_{n}\right)$ and $P$ is an $H$-projection-valued measure. Since $X$ is locally compact, then each measure $\mu_{\xi, \eta}$ is tight and regular (see [28, Theorem 7.6]), hence $P$ is regular (see Section 5). Take $f \in C_{\infty}(X, \mathbb{K})$ and form the spectral integral $E=\int_{X} f(x) P(d x)$. For each $\xi, \eta \in H$ we have $\eta^{*}(E \xi)=\int_{X} f(x) \mu_{\xi, \eta}(d x)=\eta^{*}\left(T_{f} \xi\right)$, consequently, $E=T_{f}$. In view of Equality (III) of Section 5.5 we have a regular $H$-projection-valued measures both in the case of compact and noncompact locally compact $X$.

It remains to verify the uniqueness of $P$. Suppose there exists another regular $H$-projection-valued tight measure on $X$ with the same properties. Put $\mu_{\xi, \eta}(A)=$ $\eta^{*}(P(A) \xi), \nu_{\xi, \eta}(A)=\eta^{*}(Q(A) \xi)$ for each $A \in \mathbf{L}$, where $\xi$ and $\eta \in H$. Then 
$\int_{X} f(x) \mu_{\xi, \eta}(d x)=\eta^{*}\left(T_{f} \xi\right)=\int_{X} f(x) \nu_{\xi, \eta}(d x)$ for each $f \in C_{\infty}(X, \mathbb{K})$, hence $\mu_{\xi, \eta}=\nu_{\xi, \eta}$ for each $\xi, \eta \in H$, consequently, $P(A)=Q(A)$ for each $A \in \mathbf{L}$.

COROLLARY 5.9. The relation $T_{f}=\int_{X} f(x) P(d x)$ for each $f \in C_{\infty}(X, \mathbb{K})$ sets a oneto-one correspondence between the set of all regular $H$-projection-valued tight measures $P$ on $X$ and the set of all continuous linear maps $T: C_{\infty}(X, \mathbb{K}) \rightarrow \mathscr{L}(H)$, which satisfy conditions (i), (ii), (iii) of Section 5.4.

Note 5.10. A particular case of $H=C_{\infty}(X, \mathbb{K})$ for locally compact totally disconnected Hausdorff space $X$ and $T_{f}=f$ for each $f \in C_{\infty}(X, \mathbb{K})$ can be considered independently of the given above and it is the following. Each such $f$ is a limit of a certain sequence by $n \in \mathbb{N}$ of finite sums $\sum_{j} f\left(x_{j, n}\right) \mathrm{Ch}_{V_{j, n}}(x)$, where $\left\{V_{j, n}: j \in \Lambda_{n}\right\}$ is a finite partition of $X$ into the disjoint union of $V_{j, n}$ clopen in $X, x_{j, n} \in V_{j, n}$, $\Lambda_{n} \subset \mathbb{N}$, since range $(f)$ is bounded. If we take $P(V)=\mathrm{Ch}_{V}$ for each $V \in \mathbf{L}$, then $T_{a} g=$ $\lim _{n \rightarrow \infty} \sum_{j} f\left(x_{j, n}\right) \mathrm{Ch}_{V_{j, n}}(x) g=\int_{X} f(x) P(d x) g$ for each $g \in H$, so there is the bijective correspondence between elements $a \in \mathrm{A}$ of a $C$-algebra A realised as $C_{\infty}(X, \mathbb{K})$ with $X=\mathrm{Sp}(\mathrm{A})$ and their spectral integral representations. It can be lightly seen that $P\left(V_{1} \cap V_{2}\right)=\mathrm{Ch}_{V_{1} \cap V_{2}}=\mathrm{Ch}_{V_{1}} \mathrm{Ch}_{V_{2}}=P\left(V_{1}\right) P\left(V_{2}\right)=P\left(V_{2}\right) P\left(V_{1}\right)$ for each $V_{j} \in \mathbf{L}$. If $\left\{V_{j}\right.$ : $\left.V_{j} \in \mathbf{L}, j \in \mathbb{N}\right\}$ is a disjoint family, then $P\left(\bigcup_{j} V_{j}\right) g=\mathrm{Ch}_{\cup_{j} V_{j}} g=\sum_{j} C h_{V_{j}} g=\sum_{j} P\left(V_{j}\right) g$ for each, $g \in H$. Also $P(\varnothing) H=\mathrm{Ch}_{\varnothing} H=\{0\}$ and $P(X) \mathfrak{g}=\mathrm{Ch}_{X} g=g$, for each $g \in H$. Therefore, $P$ is indeed an $H$-projection-valued tight measure.

Suppose now that $X$ is not locally compact, for example, $X=c_{0}\left(\omega_{0}, \mathbb{S}\right)$ with an infinite residue class field $k$ of a non-Archimedean infinite field $\mathbb{S}$ with nontrivial valuation. Then there are $f \in C_{\infty}(X, \mathbb{K})$ for which convergence of finite or even countable or of the cardinality card $(k)$ (which may be greater or equal to $\operatorname{card}(\mathbb{R})$ ) sums $\sum_{j} f\left(x_{j, n}\right) \mathrm{Ch}_{V_{j, n}}$ becomes a problem for a disjoint family $\left\{V_{j, n}: j\right\}$ of clopen in $X$ subsets, since $\left\|\mathrm{Ch}_{V_{j, n}}\right\|_{C(X, \mathbb{K})}=1$ for each $j$ and $n$.

THEOREM 5.11 (the non-Archimedean analog of the Stone theorem). Let A be a commutative Banach $C$-algebra over a locally compact field $\mathbb{K}$. If $P$ is a regular $H$ projection-valued tight measure on Â (see Section 5.5 and Proposition 5.7), then the equation

(i) $T_{a}=\int_{\hat{A}} \hat{a}(\phi) P(d \phi)$ for each $a \in \mathrm{A}$ defines a nondegenerate representation of $\mathrm{A}$ in $H$. Conversely, each nondegenerate representation $T$ of $\mathbf{A}$ on a Banach space $H$ determines a unique regular $H$-projection-valued tight measure $P$ on $\hat{A}$ such that (i) holds.

Proof. The right side of (i) is the spectral integral. Let $P$ be a regular $H$-projectionvalued tight measure on $\hat{A}$. By Corollary 5.9, $T^{\prime}: f \rightarrow \int_{X} f(x) P(d x)$ is a nondegenerate representation of $C_{\infty}(\hat{A}, \mathbb{K})$ on $H$. By Proposition 5.2 the map $\left.a \mapsto \hat{a}\right|_{\hat{A}}$ is a homomorphism of $\mathbf{A}$ onto a dense subset of a subalgebra of $C_{\infty}(\hat{A}, \mathbb{K})$ such that the map $T:\left.a \mapsto T^{\prime}\right|_{\left(\left.\hat{a}\right|_{\hat{A}}\right)}=\int_{\hat{A}} \hat{a} P(d \hat{a})$ is a nondegenerate representation of $\mathbf{A}$.

Conversely, let $T$ be a nondegenerate representation of $\mathbf{A}$ on $H$. Then from Sections 5.1, 5.2, and Proposition 5.2 it follows that there exists a nondegenerate representation $T^{\prime}$ of $C_{\infty}(\hat{A}, \mathbb{K})$ such that

(ii) $T_{a}=T^{\prime}{ }_{\left(\left.\hat{a}\right|_{\hat{A}}\right)}$ for each $a \in \mathrm{A}$. In view of Proposition 5.7 there exists a regular $H$-projection-valued tight measure $P$ on $\hat{A}$ satisfying the equality 
(iii) $T^{\prime} f=\int_{\hat{A}} f(x) P(d x)$ for each $f \in C_{\infty}(\hat{A}, \mathbb{K})$. Combining (ii) and (iii) we get Formula (i). Let $Q$ be another regular $H$-projection-valued tight measure which is also related to the representation $T$ by Formula (i), then

(iv) $\int_{\hat{A}} \hat{a}(x) Q(d x)=T_{a}=\int_{\hat{A}} \hat{a}(x) P(d x)$ for each $a \in$ A. Due to Proposition 5.2 $\left\{\left.\hat{a}\right|_{\hat{A}}: a \in \mathbf{A}\right\}$ is dense in $C_{\infty}(\hat{A}, \mathbb{K})$ with respect to the supremum-norm. From (iv) and Section 5, it follows that $\int_{\hat{A}} f(x) P(d x)=\int_{\hat{A}} f(x) Q(d x)$ for each $f \in$ $C_{\infty}(\hat{A}, \mathbb{K})$, consequently, by Proposition $5.7, Q=P$.

Definition 5.12. From Theorem 5.11, $P$ is called the spectral measure of the nondegenerate representation $T$ of $\mathbf{A}$.

Proposition 5.13. Let $P$ be the spectral measure of the nondegenerate representation $T$ of a commutative Banach $C$-algebra A over a locally compact field $\mathbb{K}$. If $\Omega \subset \hat{A}$ and $\Omega \in \mathbf{L}$, then

(i) $\operatorname{range}(P(\Omega))=\bigcup_{\phi \in \Omega}\left\{\xi \in H(T): T_{a} \xi=\phi(a) \xi\right.$ for each $\left.a \in \mathbf{A}\right\}$.

Proof. Relation (i) of Theorem 5.11 and the definition of the spectral integral in Proposition 5.7 show that if $\xi \in \operatorname{range} P(V)$ for each $V \in \mathbf{L}$ with $\phi \in V$, then $T_{a} \xi=$ $\phi(a) \xi$ for each $a \in \mathbf{A}$.

Conversely, suppose that $T_{a} \xi=\phi(a) \xi$ for each $a \in \mathrm{A}$. If $T^{\prime}$ is the representation of $\mathbf{A}_{t}$ isomorphic with $C_{\infty}(\hat{A}, \mathbb{K})$ and $T^{\prime}$ corresponds to $T$, then

(ii) $T^{\prime}{ }_{f} \xi=f(\phi) \xi$ for each $f \in C_{\infty}(\hat{A}, \mathbb{K})$.

Assume that $\xi \notin \operatorname{range}(P(\Omega))$ and consider a measure $\mu_{\xi, \eta}(W):=\eta^{*}(P(W) \xi)$ for $\xi$ and $\eta \in H$. There exists $\eta=\xi \neq 0$ such that $\mu_{\xi, \xi}$ is not carried by $\Omega$. Due to regularity of $\mu_{\xi, \xi}$ there exists a compact $E \subset \hat{A}, E \in \mathbf{L}, E \subset \Omega$ such that $\phi \notin E$ and $\|E\|_{\mu_{\xi, \xi}}>$ 0 . We take $f \in C_{\infty}(\hat{A}, \mathbb{K})$ which is not equal to zero everywhere on $E$ and $f(\phi)=$ 0 , since $\hat{A}$ is the completely regular topological space $T_{3.5}$ (see [9, Theorem 2.3.11]). From Formula (ii), proof of Theorem 5.11, it follows that $T^{\prime}{ }_{f} \xi=0$. By [28, Chapter 7] and Formula (VI) of Proposition 5.4 above there is an inequality: $\left\|T^{\prime}{ }_{f} \xi\right\| \geq\|f\|_{N_{\mu_{\xi, \xi}}} \geq$ $\sup _{x \in E}|f(x)| N_{\mu_{\xi, \xi}}(x)=:\left\|\left.f\right|_{E}\right\|_{N_{\mu_{\xi}, \xi}}$, where $\|f\|_{\phi}:=\sup _{x \in X}|f(x)| \phi(x)$ for $f: X \rightarrow \mathbb{K}$ and $\phi: X \rightarrow[0, \infty) ; N_{\mu}(x):=\inf _{U \in \mathbf{L}, x \in U}\|U\|_{\mu} ;\|A\|_{\mu}:=\sup \{|\mu(B)|: B \in \mathbf{L}, B \subset A\}$ for each $A \in \mathbf{L}$. If $\|\xi\|_{H}=1$, then $\left\|T^{\prime}{ }_{f} \xi\right\|=\|f\|_{N_{\mu_{\xi, \xi}}}$. We get a contradiction, consequently, $\xi \in \operatorname{range} P(\Omega)$.

5.6. Let $\mathrm{A}$ be a commutative $C$-algebra with the unit $\mathbf{1}$ over a locally compact field $\mathbb{K}$ and let $\mu$ be any regular tight measure on $\hat{A}$. Let the space $L(\hat{A}, \mu, \mathbb{K})$ be defined on the algebra $\mathbf{L}$ such that $\mathbf{L} \supset B \operatorname{co}(\hat{A})$ of $\hat{A}$ as in [28, Chapter 7], it is the completion relative to $\|f\|_{N_{\mu}}$ of the $\mathbb{K}$-linear space of all step functions, that is, finite linear combinations of characteristic functions of elements of $\mathbf{L}$.

THEOREM 5.14. The equation

(i) $\left(T_{a} f\right)(\phi)=\hat{a}(\phi) f(\phi)$ for each $a \in \mathbf{A}, f \in L(\hat{A}, \mu, \mathbb{K})$ and $\phi \in \hat{A}$ defines a nondegenerate representation $T$ of $\mathbf{A}$ on $H=L(\hat{A}, \mu, \mathbb{K})$ and the spectral measure $P$ of $T$ is given by $P(W) f=\mathrm{Ch}_{W} f$ for each $W \in \mathbf{L}$ and $f \in H$.

Proof. If $\hat{a}(\phi) \in C(\hat{A})$, then $\sup _{\phi \in \hat{A}}|\hat{a}(\phi)|<\infty$, so 


$$
\sup _{\phi \in \hat{A}}|f(\phi)||\hat{a}(\phi)| N_{\mu}(\phi) \leq\|f\|_{\mu}\|\hat{a}\|_{C(\hat{A}, \mathbb{K})},
$$

consequently, $\hat{a} f \in H$ and $T_{a} f=\int_{\hat{A}} \hat{a}(\phi) P(d \phi) f$ due to Theorem 5.11. Therefore, for $\hat{a}=\mathrm{Ch}_{W}$ we have $T_{a} f=P(W) f$ for each $W \in B \operatorname{co}(\hat{A})$. Each measure $\mu_{\xi, \eta}(W)=$ $\eta^{*}(P(W) \xi)$ has an extension from $B \operatorname{co}(\hat{A})$ on $\mathbf{L}$ due to its regularity, where $\xi$ and $\eta \in H$ (see Section 5.4). The family of such measures $\mu_{\xi, \eta}$ characterise $P$ completely, since $H$ is the Banach space of separable type over $\mathbb{K}$. Therefore, we have an extension of $P$ on $\mathbf{L}$.

5.7. From the results above it follows that $\operatorname{supp}(P) \subset \mathrm{Sp}(\mathrm{A})$. If a representation $T$ is one-to-one, that is, $\operatorname{ker} T=\{0\}$, then $T$ is called faithful.

Proposition 5.15. The kernel of a nondegenerate representation $T$ of $\mathbf{A}$ consists of $a \in \mathrm{A}$ such that $\hat{a}$ vanishes everywhere on the spectrum of T. Suppose in addition that $\mathbf{A}$ is a commutative $C$-algebra over a locally compact field $\mathbb{K}$. Then $T$ is faithful if and only if its spectrum is all of $\hat{A}$.

Proof. A condition $T_{a}=0$ is equivalent to $\int_{\hat{A}} \hat{a}(\phi) P(d \phi)=0$, which is equivalent to $\left.\hat{a}(\phi)\right|_{\hat{A}}=0$ by Theorem 5.11. Therefore, $\operatorname{ker} T=\{0\}$ is equivalent to $\operatorname{supp} T=\hat{A}$.

5.8. Fix a Banach space $H$ over a non-Archimedean complete field $\mathbb{F}$ such that $\mathbb{F} \subset$ $\mathbb{C}_{p}$. If $b \in \mathscr{L}(H)$ we write shortly $\operatorname{Sp}(b)$ instead of $\operatorname{Sp}_{\mathscr{L}(H)}(b):=\operatorname{cl}\left(\operatorname{Sp}\left(\operatorname{sp}_{\mathrm{F}}\left\{b^{n}: n=\right.\right.\right.$ $1,2,3, \cdots\})$ ) (see also [28]). If $A$ is a commutative Banach subalgebra in $\mathscr{L}(H)$, then there exists a quotient mapping $\theta: A \rightarrow A / B_{A}$, where $B_{A}$ is a closed subalgebra of $A$ such that $B_{A}=\operatorname{ker}\left(\|*\|_{\mathrm{sp}}\right)$ is the kernel of the spectral norm, $B_{A}:=\left\{x: x \in A ;\|x\|_{\mathrm{sp}}=\right.$ 0 \}. Then $\theta(A)$ is the normed algebra, $\theta(A)$ is the subalgebra of $\mathscr{L}(H) / B_{\mathscr{L}(H)}$. Choose a locally compact subfield $\mathbb{K}$ in $\mathbb{F}$.

THEOREM 5.16 (spectral theorem for operators). Let $b \in \mathscr{L}(H)$. Then there exists a unique $H$-projection-valued tight measure $P$ on $\mathbb{K}$ with values in $\mathscr{L}(H)$ with the following properties:

(i) the closed support $D$ of $P$ is bounded in $\mathbb{K}$;

(ii) $\theta(b)=\int_{\mathbb{K}} x P(d x)$; also $b=\lambda_{b} V \int_{\mathbb{K}} x P(d x)$, where $V$ is a continuous operator from $H$ onto its closed $\mathbb{K}$-linear subspace such that $\left|\pi_{\mathbb{K}}\right| \leq\|V\| \leq\left|\pi_{\mathbb{K}}\right|^{-1}, \pi_{\mathbb{K}} \in \mathbb{K}$, $\left|\pi_{\mathbb{K}}\right|=\max \{|x|: x \in \mathbb{K},|x|<1\} ;\left|\lambda_{b}\right|=\|b\|, \lambda_{b} \in \mathbb{F} ; V$ is an isometry of $H$ onto its closed $\mathbb{K}$-linear subspace for $\mathbb{K}=\mathbb{F}$;

(iii) if $\mathbb{K}=\mathbb{F}$, then $D=\operatorname{Sp}(a)$, where $a$ is an auxiliary operator defined by $V$ and $b$. Moreover, if $S$ is a family of commuting operators, $S \subset \mathscr{L}(H)$, then there exists a unique $H$-projection-valued tight measure $P$ on a locally compact subset $X \subset$ $B(\mathbb{K}, 0,1,)^{\gamma}$ such that for each $b \in S$ there exists a unique $f_{b} \in C_{\infty}(X, \mathbb{F})$ for which $\theta(b)=\int_{X} f_{b}(x) P(d x), b=V \int_{X} f_{b}(x) P(d x)$ and $V$ as above, where $\operatorname{cl} X=X \cup\{0\}$ is compact.

Proof. If $\|b\|_{\mathrm{sp}}=0$, then $\operatorname{Sp}(b)=\varnothing$ and this case is trivial with $P(\varnothing)=0$. In the case of the locally compact field $\mathbb{K}$ and $H$ over $\mathbb{K}$ we can take $W:=\operatorname{cl}(b(H))$ and $b=V a$, where $V$ is an operator such that $V(H)=W,\left.V\right|_{W}: W \rightarrow W$ is an isometry, $V(H \ominus W)=$ 
$\{0\}, a$ is an operator of $H$ onto $H$ such that $\|a\|_{\text {sp }}>0$ and $a$ is representable as a convergent series of projectors in some basis of $H$, that can be shown by transfinite induction (see [26]). In this case we get (ii). Analogously for commuting algebras of operators.

The field $\mathbb{E}$ can be considered as the Banach space over $\mathbb{K}$. This means that $\mathbb{F}$ supplied with the linear structure over $\mathbb{K}$ is isomorphic with $c_{0}(\beta, \mathbb{K})$ and a corresponding ordinal $\beta$, since $\mathbb{K}$ is locally compact and hence spherically complete (see [28, Theorems 5.13 and 5.16]). This isomorphism $\chi: \mathbb{F} \rightarrow c_{0}(\beta, \mathbb{K})$ may be nonisometrical. The isomorphism $\chi$ generates the isomorphism of $H$ considered as the Banach space $H_{\mathbb{K}}$ over $\mathbb{K}$ with $c_{0}\left(\alpha_{\mathbb{K}}, \mathbb{K}\right), \chi: H_{\mathbb{K}} \rightarrow c_{0}\left(\alpha_{\mathbb{K}}, \mathbb{K}\right)$ with the corresponding ordinal $\alpha_{\mathbb{K}}$. This isomorphism is $\mathbb{K}$-linear and it produces an injective continuous $\mathbb{K}$-linear embedding $\chi^{*}: \mathscr{L}(H) \rightarrow \mathscr{L}\left(c_{0}\left(\alpha_{\mathbb{K}}, \mathbb{K}\right)\right)$ with continuous $\left.\left(\chi^{*}\right)^{-1}\right|_{\chi^{*}(\mathscr{L}(H))}$. The embedding $\chi^{*}$ is given by the following formula: $\chi^{*}(a) y:=\chi a x^{-1} y$ for each $a \in \mathscr{L}(H)$ and $y \in c_{0}\left(\alpha_{\mathbb{K}}, \mathbb{K}\right)$. This is the well-known construction of the contraction of a scalar field for a Banach space. In the particular case of $H=\mathbb{F}^{n}$ with $n \in \mathbb{N}$ and if $\mathbb{F}$ is a finite algebraic extension of $\mathbb{K}$ to each $a \in \mathscr{L}(H)$, there corresponds a finite $n \times n$ matrix, hence $\chi^{*}(a) \in \mathscr{L}\left(\mathbb{K}^{\beta n}\right)$.

Suppose there is a representation of a $C$-algebra $C_{\infty}(X, \mathbb{F})$ with the help of a $c_{0}\left(\alpha_{\mathbb{K}}\right.$, $\mathbb{K})$-projection-valued tight measure $P$ on a locally compact subset $X$ in $\mathbb{K}^{\gamma}$. Then $\left(\chi^{*}\right)^{-1}$ produces from $P$ an $H$-projection-valued tight measure $P_{\mathbb{F}}$, since

$$
\left(\chi^{-1} P(V) \chi\right)\left(\chi^{-1} P(W) \chi\right)=\chi^{-1} P(V \cap W) \chi,
$$

for each $V$ and $W \in \mathbf{L}$. Consequently,

$$
\chi^{-1} \int_{X} g(x) P(d x) \chi z=\int_{X}\left(x^{-1} g(x) \chi\right)\left(x^{-1} P(d x) \chi\right) z,
$$

for each $z \in H$ and $f \in C_{\infty}(X, \mathbb{K})$. Therefore, if $\chi^{*}(a)=\int_{X} g_{a}(x) P(d x)$, then $a=$ $\int_{X} f_{a}(x) P_{\mathbb{E}}(d x)$, where $g_{a} \in C_{\infty}(X, \mathbb{K})$ and hence $\chi^{*}\left(g_{a}\right)=: f_{a} \in C_{\infty}(X, \mathbb{F})$ such that $f_{a}=g_{a}$, since the restriction of $X$ on $\mathbb{K}$ embedded into $\mathbb{E}$ is the identity $\mathbb{K}$-linear mapping.

It follows that instead of $b$ or $S$, it is sufficient to consider $\chi^{*}(b)$ or $\chi^{*}(S)$. Denote $\chi^{*}(b)$ and $\chi(S)$ simply by $b$ and $S$, respectively. The operator $b$ or the family $S$ generates a commutative subalgebra $A$ of $\mathscr{L}\left(H_{\mathbb{K}}\right)$ generated by $\operatorname{sp}_{\mathbb{K}}\left\{b^{n}: 0 \leq n \in \mathbb{Z}\right\}$ or by $\operatorname{sp}_{\mathbb{K}}\left\{a_{1}^{m_{1}} \cdots a_{n}^{m_{n}}: a_{j} \in S, n \in \mathbb{N}, 0 \leq m_{j} \in \mathbb{Z} ; j=1, \ldots, n\right\}$, where $b^{0}:=1$. It has a completion A relative to the spectral norm $\|*\|_{\text {sp }}$ by [28, Chapter 6]. This A in view of [28, Theorem 6.15 and Corollaries $6.16,6.17]$ is a Banach $C$-algebra $C(\operatorname{Sp}(\mathbf{A}), \mathbb{K})$, since $\mathbf{1} \in \mathbf{A}$ and $\operatorname{Sp}(A)$ is compact such that $C(E, \mathbb{K})$ is isomorphic with $C_{\infty}(E, \mathbb{K})$ for compact $E$.

For $S=\{b\}$ each $\phi \in A^{+}$is completely defined by $\phi(b)$, thus the map $\phi \mapsto \phi(b)$ is continuous and one-to-one, consequently, it is a homeomorphism from the compact space $\operatorname{Sp}(\mathbf{A})$ onto the compact subset $\mathrm{Sp}(b)$ of $\mathbb{K}$. Therefore, we identify $\operatorname{Sp}(\mathbf{A})$ with $\operatorname{Sp}(b)$. So the Gelfand transform of $b$ becomes the identity function on $\operatorname{Sp}(b)$. Thus by Theorem 5.11 the identity representation of $\mathbf{A}$ in $H$ gives rise to the $H$-projectionvalued tight measure $P$ on $\operatorname{Sp}(b)$ such that $\theta(b)=\int_{D} x P(d x)$ and $b=\lambda_{b} V \int_{D} x P(d x)$. Since the identity representation is faithful, then Proposition 5.15 shows that the closed support $D$ of $P$ is homeomorphic to $\operatorname{Sp}(b)$ (up to the mapping $\left.\chi\right|_{\mathbb{K}}$ ). Extending 
$P$ to $\mathbf{L}$ by setting $P(W)=P((W) \cap \operatorname{Sp}(b))$, we obtain a tight projection-valued measure on $\mathbb{K}$ satisfying (i), (ii), (iii) of Section 5.4 .

In the case of the family $S$ take $X=\operatorname{Sp}$ (A) such that, [9, Theorem 2.3.20], about a diagonal mapping we can choose $\mathbf{a} \leq \operatorname{card}(\gamma) \leq \operatorname{card}\left(\mathbf{A}^{+}\right)$while embedding $X \hookrightarrow$ $B(\mathbb{K}, 0,1)$, where $\mathbf{a}$ is the minimal cardinality of a family of subsets in $\mathbf{A}^{+}$separating points of A. In view of [28, Section 6.2], $\operatorname{cl}(X)=X \cup\{0\}$.

To show that $P$ is uniquely determined by Theorem 5.16(i) and (ii), let $P^{\prime}$ be another $H$-projection-valued tight measure on $\mathbb{K}$ satisfying Theorem 5.16(i) and (ii) and let $E$ be a compact subset of $\mathbb{K}$ containing the supports of both $P$ and $P^{\prime}$. Consider two representations $T: f \mapsto \int_{E} f(x) P(d x)$ and $T^{\prime}: f \mapsto \int_{E} f(x) P^{\prime}(d x)$ of $C(E, \mathbb{K})$. If $w$ and $e$ are the identity function $w(x)=x$ and the constant function $e(x)=1$ for each $x \in E$, respectively, then condition (ii), satisfied by both $P$ and $P^{\prime}$, shows that $T_{w}=T^{\prime}{ }_{w}=b$ and also $T_{e}=T_{e}^{\prime}=1$. By the Kaplansky theorem $w$ and $e$ generate $C(E, \mathbb{K})$ as a $C$-algebra [25, 28], hence $T^{\prime}=T$ and by the uniqueness statement of Theorem 5.11 we have $P^{\prime}=P$.

5.9. The $P$ of the above theorem is called the spectral measure of the operator $b$ or of a family $S$. In particular, $S$ may be a commutative subalgebra of $\mathscr{L}(H)$. Evidently, each nilpotent operator $v$ in $\mathscr{L}(H)$ has $\|v\|_{\text {sp }}=0$. If $v \in \mathscr{L}_{0}(H)$ has $e_{j}^{*}\left(u e_{i}\right)=0$ for each $j \leq i$, then $v$ is nilpotent. Therefore, $\theta\left(\mathscr{L}_{0}(H)\right)$ is isomorphic with the algebra of diagonal operators on $H$. Its spectrum was found in Section 4.

NoTE 5.17. It is an interesting property of the non-Archimedean case that a condition of a normality of an operator $b$ is not necessary in Theorem 5.16 apart from the classical case. It is not so surprising if we recall that orthogonality in the nonArchimedean case is quite different from the classical case. For example, two vectors $(1,0)$ and $(1,1)$ are orthonormal in $\mathbb{K}^{2}$, but are not orthogonal in $\mathbb{C}^{2}$.

\section{REFERENCES}

[1] S. Albeverio, J. M. Bayod, C. Perez-Garcia, R. Cianci, and A. Khrennikov, Non-Archimedean analogues of orthogonal and symmetric operators and p-adic quantization, Acta Appl. Math. 57 (1999), no. 3, 205-237.

[2] S. Albeverio, R. Cianci, and A. Khrennikov, On the spectrum of the p-adic position operator, J. Phys. A 30 (1997), no. 3, 881-889.

[3] V. G. Berkovich, Spectral Theory and Analytic Geometry over Non-Archimedean Fields, Mathematical Surveys and Monographs, vol. 33, American Mathematical Society, Rhode Island, 1990.

[4] B. Diarra, Ultraproduits ultramétriques de corps valués, Ann. Sci. Univ. Clermont-Ferrand II Math. (1984), no. 22, 1-37 (French).

[5] _ Remarques sur les matrices orthogonales [resp. symétriques] à coefficients padiques, Ann. Sci. Univ. Clermont-Ferrand II Math. (1990), no. 26, 31-50 (French).

[6] _ An operator on some ultrametric Hilbert spaces, J. Anal. 6 (1998), 55-74.

[7] N. Dunford and J. T. Schwartz, Linear Operators. I. General Theory, Interscience Publishers, New York, 1958.

[8] _ Linear Operators. Part II: Spectral Theory. Self Adjoint Operators in Hilbert Spaces, Interscience Publishers John Wiley \& Sons New York-London, 1963.

[9] R. Engelking, Obshchaya Topologiya, Mir, Moscow, 1986 (Russian).

[10] A. Escassut, The ultrametric spectral theory, Period. Math. Hungar. 11 (1980), no. 1, 7-60.

[11] _ _ Analytic Elements in p-adic Analysis, World Scientific, Singapore, 1985. 
[12] A. Escassut and N. Mainetti, Spectral semi-norm of a p-adic Banach algebra, Bull. Belg. Math. Soc. Simon Stevin 5 (1998), no. 1, 79-91.

[13] J. M. G. Fell and R. S. Doran, Representations of *-Algebras, Locally Compact Groups, and Banach *-Algebraic Bundles. Vol. 1, Academic Press, Massachusetts, 1988.

[14] _ Representations of *-Algebras, Locally Compact Groups, and Banach *-Algebraic Bundles. Vol. 2, Academic Press, Massachusetts, 1988.

[15] F. R. Gantmaher, The Theory of Matrices, Chelsea Publ. Comp., New York, 1977.

[16] - The Theory of Matrices, Nauka, Moscow, 1988.

[17] M. Goto and F. D. Grosshans, Semisimple Lie Algebras, Marcel Dekker, New York, 1978.

[18] L. Gruson, Théorie de Fredholm p-adique, Bull. Soc. Math. France 94 (1966), 67-95 (French).

[19] E. Hewitt and K. A. Ross, Abstract Harmonic Analysis. Vol. I, Springer-Verlag, Berlin, 1979.

[20] H. A. Keller and A. H. Ochsenius, Algebras of bounded operators on nonclassical orthomodular spaces, Int. J. Theoret. Phys. 33 (1994), no. 1, 1-11.

[21] _ B B Bunded operators on non-Archimedian orthomodular spaces, Math. Slovaca 45 (1995), no. 4, 413-434.

[22] A. Khrennikov, Non-Archimedean Analysis: Quantum Paradoxes, Dynamical Systems and Biological Models, Kluwer Academic Publishers, Dordrecht, 1997.

[23] M. A. Naĭmark, Normirovannye Koltsa, 2nd revised ed., Izdat. Nauka, Moscow, 1968 (Russian).

[24] H. Ochsenius and W. H. Schikhof, Banach spaces over fields with an infinite rank valuation, $p$-adic functional analysis (Poznań, 1998), Dekker, New York, 1999, pp. 233-293.

[25] W. H. Schikhof, Ultrametric Calculus, Cambridge University Press, Cambridge, 1984.

[26] M. van der Put, The ring of bounded operators on a non-Archimedean normed linear space, Nederl. Akad. Wetensch. Proc. Ser. A 30 (1968), 260-264.

[27] _ Difference equations over p-adic fields, Math. Ann. 198 (1972), 189-203.

[28] A. C. M. van Rooij, Non-Archimedean Functional Analysis, Marcel Dekker, New York, 1978.

[29] A. C. M. van Rooij and W. H. Schikhof, Non-archimedean commutative $C^{*}$-algebras, Indag. Math. 35 (1973), 381-389.

[30] M. M. Vishik, Non-archimedean spectral theory, J. Sov. Math. 30 (1985), 2513-2554.

S. Ludkovsky: Theoretical Department, Institute of General Physics, 38, VAVilov STREeT, Moscow, 119991, Russia

E-mail address: 1udkovsk@fp1.gpi.ru

B. Diarra: Laboratoire de Mathématiques Pures, Complexe SCientifique des CÉZEAuX, 63177 AUBIÈRE, FRANCE

E-mail address: Bertin.Diarra@math. univ-bpclermont.fr 


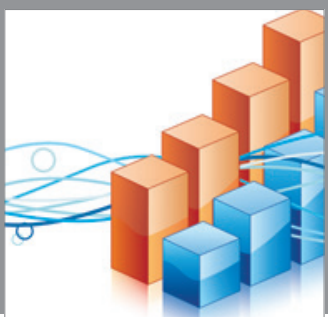

Advances in

Operations Research

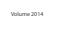

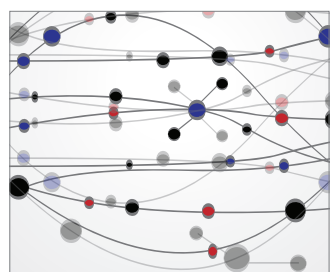

\section{The Scientific} World Journal
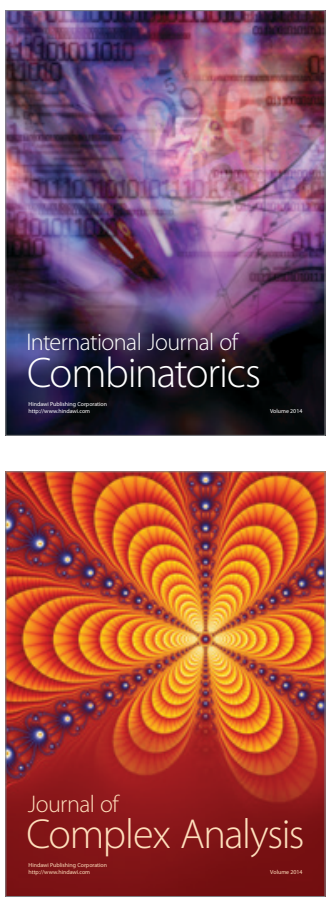

International Journal of

Mathematics and

Mathematical

Sciences
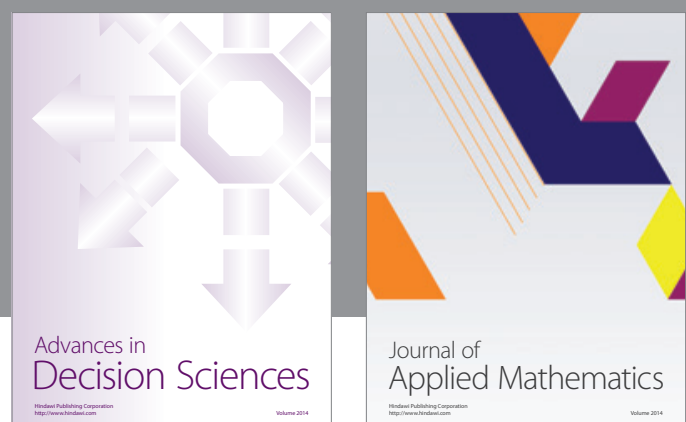

Journal of

Applied Mathematics
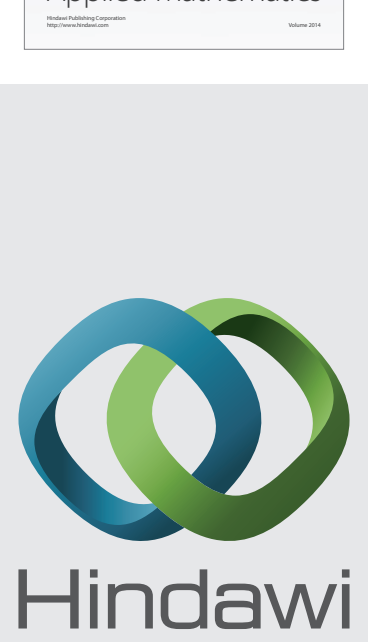

Submit your manuscripts at http://www.hindawi.com
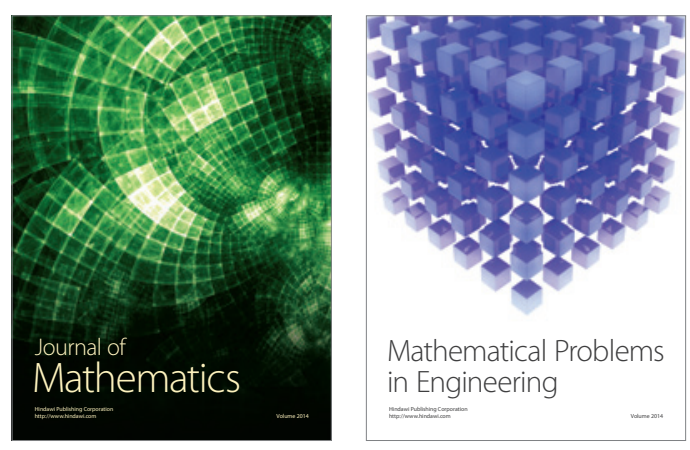

Mathematical Problems in Engineering
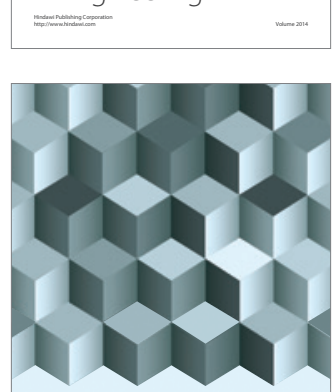

Journal of

Function Spaces
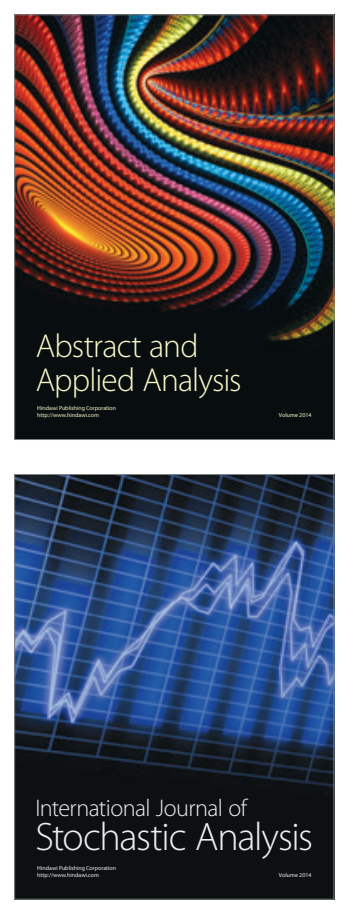

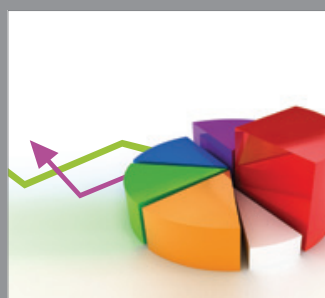

ournal of

Probability and Statistics

Promensencen
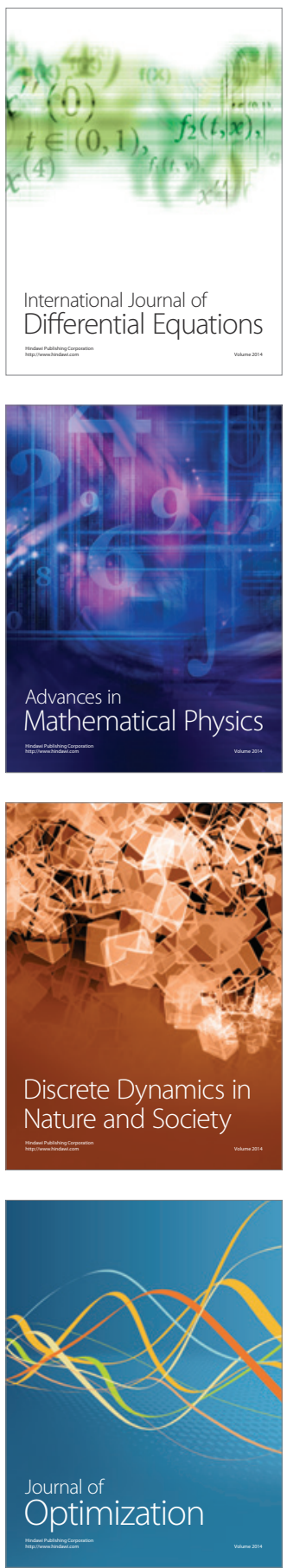